\title{
\#USGS
}

Eusches

National Wildilfe Health Center

\section{Entianced surveillance Strategies for Detecting and Monitoring Chronic Wasting Disease in Free-Ranging Cenvios}

Openatile Repo:t $2012-1036$

U.S. Department of the Interior

U.S. Geological Survey 
Front and back cover photographs by Dr. Daniel P. Walsh 


\section{Enhanced Surveillance Strategies for Detecting and Monitoring Chronic Wasting Disease in Free-Ranging Cervids}

Edited by Dr. Daniel P. Walsh

Chapter 1

Risk Factors for Chronic Wasting Disease: A Deer Is Not a Deer

Is Not a Deer

By Daniel P. Walsh, Ph.D., and Michael D. Samuel, Ph.D.

Chapter 2

Disease Surveillance: Incorporating Available Information to Enhance Disease-Detection Efforts

By Daniel P. Walsh, Ph.D., and David L. Otis, Ph.D.

Chapter 3

Monitoring Surveillance: Disease Investigations in Chronic Wasting Disease Positive Areas

By Julie A. Langenberg, VMD, Daniel J. O'Brien, DVM, Ph.D., Daniel P. Walsh, Ph.D., Lynn H. Creekmore, DVM, Jenny G. Powers, DVM, Ph.D., and Michael W. Miller, DVM, Ph.D.

National Wildlife Health Center

Open-File Report 2012-1036 


\title{
U.S. Department of the Interior \\ KEN SALAZAR, Secretary \\ U.S. Geological Survey \\ Marcia K. McNutt, Director
}

\author{
U.S. Geological Survey, Reston, Virginia: 2012
}

For more information on the USGS - the Federal source for science about the Earth, its natural and living resources, natural hazards, and the environment, visit http://www.usgs.gov or call 1-888-ASK-USGS.

For an overview of USGS information products, including maps, imagery, and publications, visit http://www.usgs.gov/pubprod

To order this and other USGS information products, visit http://store.usgs.gov

Any use of trade, product, or firm names is for descriptive purposes only and does not imply endorsement by the U.S. Government.

Although this report is in the public domain, permission must be secured from the individual copyright owners to reproduce any copyrighted materials contained within this report.

Suggested citation:

Walsh, D.P.,ed., 2012, Enhanced surveillance strategies for detecting and monitoring chronic wasting disease in freeranging cervids: U.S. Geological Survey Open-File Report 2012-1036. 42 p. 


\section{Preface}

In 2003, Surveillance Strategies for Detecting CWD in Free-ranging Deer and Elk was made available to wildlife managers as they developed or enhanced their chronic wasting disease (CWD) programs. The document was the product of a 2002 meeting hosted by the U.S. Geological Survey-National Wildlife Health Center (USGS-NWHC). Although the guidelines were stateof-the-art when published, wildlife managers more recently have expressed the need for more efficient surveillance strategies based on scientific information developed since 2003.

At the request of the Association of Fish and Wildlife Agencies (AFWA), Bryan Richards of the USGS-NWHC led a 2008 meeting of wildlife health professionals, statisticians, and biometricians to update CWD surveillance strategies. The following chapters on CWD risk factors, surveillance, and monitoring were drafted by meeting participants and others in 2008. The intent of these chapters is to provide wildlife professionals with knowledge regarding recent developments in the arena of CWD surveillance, as well as present the collective experience of meeting participants in constructing, managing, and researching CWD surveillance techniques and programs. It is hoped that this information will aid managers in implementing more efficient surveillance and monitoring programs.

In 2010, AFWA formed a working group charged with providing members with updated surveillance strategies in response to further erosion of public support and financial support for CWD surveillance and monitoring. The AFWA CWD Working Group reviewed the three chapters, found them to be an excellent resource for wildlife managers. 



\section{Executive Summary}

The purpose of this document is to provide wildlife management agencies with the foundation upon which they can build scientifically rigorous and cost-effective surveillance and monitoring programs for chronic wasting disease (CWD) or refine their existing programs. The first chapter provides an overview of potential demographic and spatial risk factors of susceptible wildlife populations that may be exploited for CWD surveillance and monitoring. The information contained in this chapter explores historic as well as recent developments in our understanding of CWD disease dynamics. It also contains many literature references for readers who may desire a more thorough review of the topics or CWD in general. The second chapter examines methods for enhancing efforts to detect CWD on the landscape where it is not presently known to exist and focuses on the efficiency and cost-effectiveness of the surveillance program. Specifically, it describes the means of exploiting current knowledge of demographic and spatial risk factors, as described in the first chapter, through a two-stage surveillance scheme that utilizes traditional design-based sampling approaches and novel statistical methods to incorporate information about the attributes of the landscape, environment, populations and individual animals into CWD surveillance activities. By accounting for these attributes, efficiencies can be gained and cost-savings can be realized. The final chapter is unique in relation to the first two chapters. Its focus is on designing programs to monitor CWD once it is discovered within a jurisdiction. Unlike the prior chapters that are more detailed or prescriptive, this chapter by design is considerably more general because providing comprehensive direction for creating monitoring programs for jurisdictions without consideration of their monitoring goals, sociopolitical constraints, or their biological systems, is not possible. Therefore, the authors draw upon their collective experiences implementing disease-monitoring programs to present the important questions to consider, potential tools, and various strategies for those wildlife management agencies endeavoring to create or maintain a CWD monitoring program. Its intent is to aid readers in creating efficient and cost-effective monitoring programs, while avoiding potential pitfalls. It is hoped that these three chapters will be useful tools for wildlife managers struggling to implement efficient and effective CWD disease management programs. 



\section{Contents}

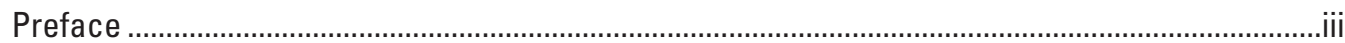

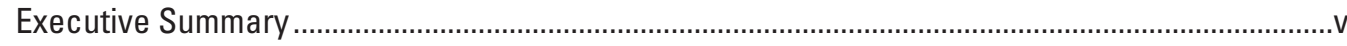

Chapter 1

Risk Factors for Chronic Wasting Disease: A Deer Is Not a Deer Is Not a Deer

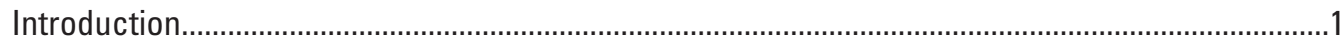

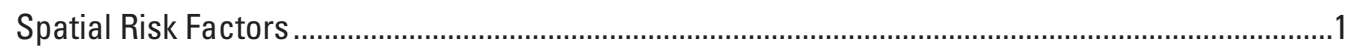

Demographic Risk Factors ......................................................................................................

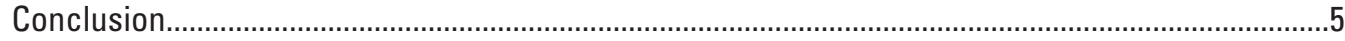

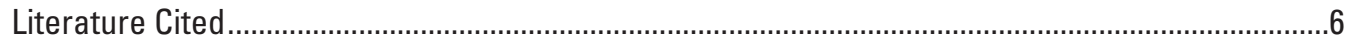

Chapter 2

Disease Surveillance: Incorporating Available Information to Enhance Disease-Detection Efforts

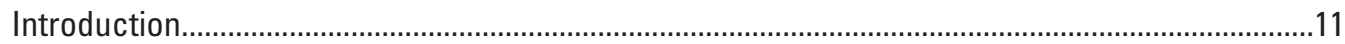

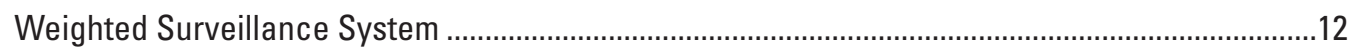

Probabilistic Sampling Designs for Selection of Sampling Units-Clustering and

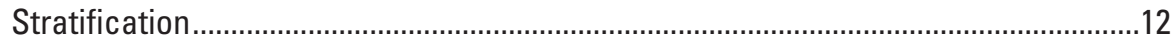

Unequal Probability Sampling and Risk Factors..................................................................

Simulation Studies —Spatial Distribution of Samples ...........................................................13

Selection of Population Elements from within Sampling Units—No Risk Data ......................16

Selection of Population Elements from within Sampling Units-Risk Data Available ..............17

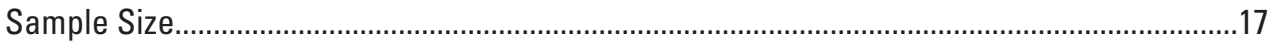

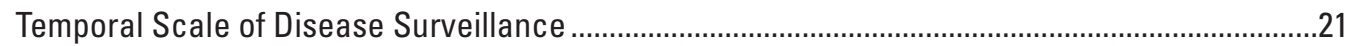

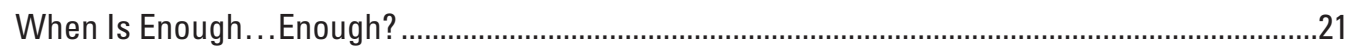

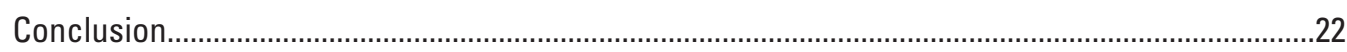

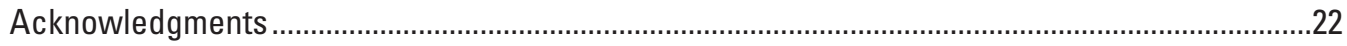

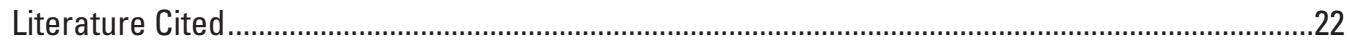

Chapter 3

Monitoring Surveillance: Disease Investigations in Chronic Wasting Disease-Positive Areas

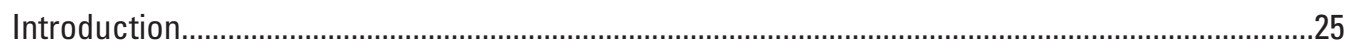

Initial Assessment of a New Chronic Wasting Disease Focus: Evaluating Intensity

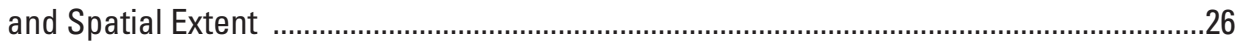

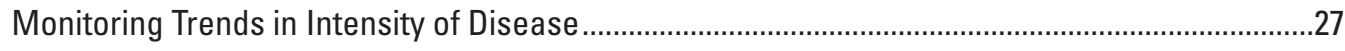

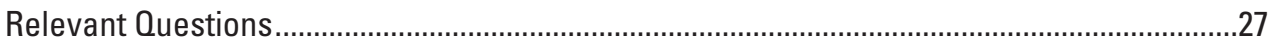

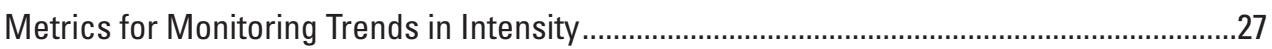

Number of Chronic Wasting Disease-Positive Animals...............................................28

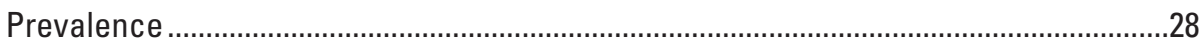

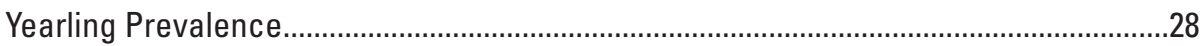

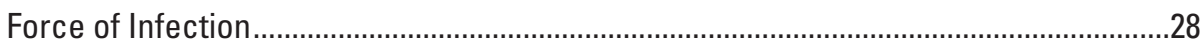

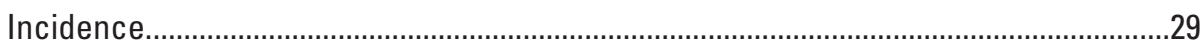

Strategies and Approaches for Intensity Monitoring ……..............................................2

Where and How Many to Sample for Intensity Monitoring.....................................................31 
Chapter 3

\section{Monitoring Surveillance: Disease Investigations in Chronic Wasting Disease-Positive Areas-Continued}

Challenges with Intensity Monitoring

Monitoring Trends in Spatial Distribution ………….................................................................32

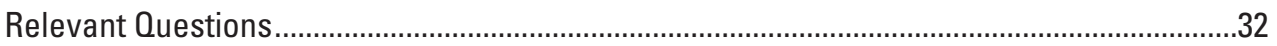

Considerations in Monitoring Spatial Changes ..................................................................33

Metrics for Monitoring Trends in Spatial Distribution ............................................................33

Strategies and Approaches for Monitoring Surveillance .....................................................34

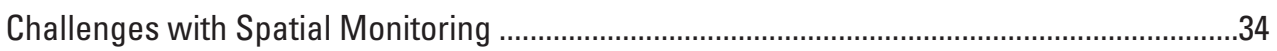

Research and Chronic Wasting Disease Monitoring Surveillance ................................................36

Research That Could Improve Planning of Monitoring Surveillance .....................................36

Research Ideas to Consider Incorporating With a Monitoring Plan.......................................36

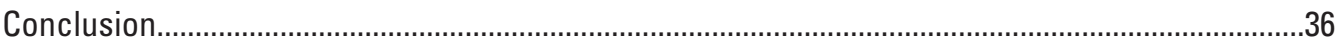

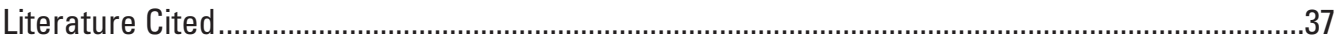

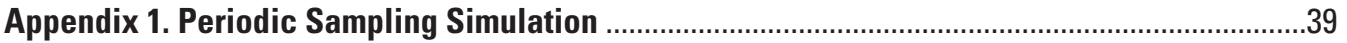

\section{Figures}

1. Diagram showing landscape used in sampling simulations

2. Graphs showing $A$, Examples of distributions of sampling locations used in the simulations. $B$, The probability of detecting chronic wasting disease on a landscape as a function of sample size and dispersion of samples.

3. Map showing sampling regions for detecting changes in spatial distribution of chronic wasting disease in Wisconsin during 2006-7

1-1. Graphs showing comparison of mean chronic wasting disease prevalence estimates and their measures of precision between $A$, Scenario $A$ with continuous disease monitoring and $B$, Scenario $B$ with periodic sampling generated during 10,000 simulation repetitions...

1-2. Graphs showing comparison of proportion of 10,000 simulation repetitions where the correct underlying disease model was selected based on Akaike's Information Criterion (AIC) between a continuous sampling regime (Scenario A) and a periodic sampling scheme (Scenario B)

1-3. Graphs showing comparison of mean trend estimates and mean, standard 95-percent confidence bounds for models where the correct disease model was selected by using Akaike's Information Criterion (AIC) between $A$, Scenario $A$ with continuous disease monitoring and $B$, Scenario $B$ with periodic sampling (generated during 10,000 simulation repetitions) 


\section{Tables}

1. Estimated mean hazard ratios, associated standard deviations,

Markov Chain Monte Carlo (MCMC) errors, and quantiles for various demographic groups of mule deer (Odocoileus hemionus) based on samples collected from chronic wasting disease-positive areas in Colorado during 2003-6

2. Estimated mean hazard ratios, associated standard deviations, Markov Chain Monte Carlo (MCMC) errors, and quantiles for various demographic groups of elk (Cervus elaphus) based on samples collected from chronic wasting disease-positive areas in Colorado during 2003-6

3. The "real weights" that account for uncertainty in the estimates of the hazard ratios and associated number of samples $(\mathrm{N})$ needed to reach a 95-percent confidence that an area of interest is "disease-free" if the prevalence is less than or equal to 0.01 for mule deer (Odocoileus hemionus) from chronic wasting disease-positive areas in Colorado during 2003-6.....

4. The "real weights" that account for uncertainty in the estimates of the hazard ratios and associated number of samples $(\mathrm{N})$ needed to reach a 95-percent confidence that an area of interest is "disease-free" if the prevalence is less than or equal to 0.01 for elk (Cervus elaphus) from chronic wasting disease-positive areas in Colorado during 2003-6 



\title{
Chapter 1 Risk Factors for Chronic Wasting Disease: A Deer Is Not a Deer Is Not a Deer
}

\author{
By Daniel P. Walsh, Ph.D. ${ }^{1}$, and Michael D. Samuel, Ph.D. ${ }^{2}$
}

\section{Introduction}

Risk factors are attributes of the landscape, environment, populations, and individual animals associated with a greater probability of chronic wasting disease (CWD) occurring in a target region or target population. Identifying and understanding the importance of various risk factors is a critical step in designing both CWD surveillance and monitoring strategies. This is particularly critical for CWD, because most areas have low disease prevalence that is difficult to detect, and the disease is distributed heterogeneously over the landscape. Current information suggests that CWD occurrence and prevalence varies among geographic areas (states), among regions within states, and occurs in clusters of affected animals within these regions. As a result, surveillance to detect CWD without considering potential risk factors is likely to be inefficient and less effective, and failure to understand risk factors may bias disease metrics, such as population prevalence or detection probability, used in CWD surveillance and monitoring efforts.

The motivation behind using risk factors in surveillance and monitoring programs is to increase efficiency given existing or reduced resources (Stark and others, 2006). In other words, by utilizing our knowledge of various risk factors and incorporating this information into surveillance and monitoring activities, we can capitalize on all available information concerning the disease; consequently, the result is more efficient use of resources. The idea of using risk factors to enhance surveillance and monitoring efforts is not novel and has been successfully employed in surveillance for other diseases, including other transmissible spongiform encephalopathies (TSEs). For example, surveillance programs for bovine spongiform encephalopathy (BSE) in the European Union have exploited risk factors by focused sampling of animals that were most likely to be infected (Doherr and others, 2001; Wilesmith and others, 2004). Thus, CWD surveillance and monitoring programs can also be enhanced by exploiting existing knowledge regarding risk factors.

We describe below two classes of risk factors: spatial and demographic risk factors. Within these classes, we present various risk factors associated with CWD and detail our current knowledge or lack thereof for these factors. Knowledge of CWD risk factors has increased substantially since 2003, but despite advances in understanding, knowledge remains limited. Continued research to enhance understanding of risk factors is needed to improve the efficiency of surveillance programs and understanding of CWD epidemiology. Our purpose herein is to provide the current information regarding risk factors, which may be exploited for surveillance and monitoring activities, as well as elucidate knowledge gaps.

\section{Spatial Risk Factors}

Spatial risk factors are attributes associated with the location, arrangement and composition of landscape, environmental, and animal characteristics that increase the probability of disease (CWD) occurrence and prevalence. Establishing the presence (or absence) and level of spatial risk factors in a target region is fundamental for focusing attention and allocating resources in any surveillance or monitoring program. This is particularly important for CWD, because in most areas disease may only occur at a low prevalence and is heterogeneously distributed over the landscape. As a result, identifying areas or populations at higher risk based on knowledge of spatial attributes can help focus surveillance and monitoring activities, reduce costs, and increase the likelihood of early disease detection. CWD spatial risk factors can generally be divided into two groups: those related to introduction of disease into a new area or target population and those specific to the rate of disease spread through the area or population. At the current

${ }^{1}$ Colorado Division of Wildlife, currently affiliated with the U.S. Geological Survey, National Wildlife Health Center.

${ }^{2}$ U.S. Geological Survey, Wisconsin Cooperative Fish and Wildlife Research Unit. 
Enhanced Surveillance Strategies for Detecting and Monitoring Chronic Wasting Disease in Free-Ranging Cervids

time, our knowledge of these spatial risk factors is limited and a better understanding would improve the efficiency and rigor of surveillance and monitoring programs.

Current information suggests that CWD occurrence and prevalence can vary at multiple spatial scales: among geographic areas (states or provinces), among regions within states/provinces, or in clusters within these regions (Samuel and others, 2003). In many cases it appears that the length of time CWD has been present in a specific area may be associated with spatial distribution and prevalence (Joly and others, 2006; Heisey and others, 2010). For example, in Colorado and Wyoming, CWD appears to be fairly widely distributed; however, prevalence varies considerably among deer management units within those states (Conner and Miller, 2004; Miller and Conner, 2005; Wyoming Game and Fish Department, 2010). In Illinois and Wisconsin, where CWD was likely introduced more recently, CWD follows distinct patterns of spatial spread from the likely areas of introduction (Joly and others, 2003; Joly and others, 2006; Osnas and others, 2009; Heisey and others, 2010; Shelton and McDonald, 2011). Spatial distribution of CWD is also likely influenced by seasonal movement patterns, dispersal, and home range use of cervids (Conner and Miller, 2004; Farnsworth and others, 2006). Recent studies using landscape genetics suggest that rates of deer dispersal, landscape features that affect migration behavior, and dispersal by male white-tailed deer (Odocoileus virginianus) may be a useful predictors of spatial risk (Blanchong and others, 2008; Kelly and others, 2010; Cullingham, Nakada, and others, 2011), but the time scale required for CWD spread is not currently understood. At a local scale, transmission of disease among individual animals appears to be associated with local interaction among animals within the same or adjacent home range areas (Farnsworth and others, 2006; Osnas and others, 2009; Grear and others, 2010; Kelly and others, 2010; Cullingham, Nakada, and others, 2011) and especially for females in the same social group (Grear and others, 2010). These local interactions can lead to increased prevalence within localized areas and fine scale variability in CWD spatial patterns across the landscape. As a result in free-ranging cervid populations, detection of a CWD-infected individual strongly suggests an increased risk of infection for animals using similar or adjacent areas, although the spatial extent of risk may depend on the time CWD has been present and the prevalence of disease. Thus, it is clear that detection of a positive individual in an area is an important spatial risk factor that should be exploited when conducting CWD surveillance and monitoring.

In addition to locations of known CWD-positive individuals, other spatial risk factors related to CWD exposure should be considered. For example, the risk of free-ranging animals being exposed to CWD is likely greater in areas where captive cervid facilities have or had CWD-positive animals. Current evidence indicates that CWD infection rates are much higher in captive facilities than in wild populations (Keane and others, 2008), and perhaps this is driven by environmental contamination (Miller and others, 2006). This higher rate of infection in captive animals can increase the risk of disease exposure to surrounding wild populations. Furthermore, movement of infectious animals, carcasses, or other materials across the landscape, naturally or with human assistance, likely increases the risk to uninfected populations. The frequent movement of farmed elk (Cervus elaphus) and deer between production facilities, the concentration of infected animals on some facilities, and the possibility of their escape into the wild increases the risk of spreading CWD to uninfected populations of free-ranging animals. Because the infectious prions may persist in the environment for long periods, the introduction of either captive or free-ranging uninfected animals into a contaminated environment could increase their risk of infection. For example, locations from which sheep have been removed may remain contaminated with scrapie agent for more than 15 years (Georgsson and others, 2006). In a similar manner, translocation of cervids from areas that have not been documented to be CWD-free could pose a risk of disease introduction. In this situation, the risk of introduction is likely related to the probability of infected animals being moved and their ability to spread CWD to other susceptible animals or into the environment. Thus, surveillance on and around cervid farms or free-ranging populations that have received animals from known CWD areas and bordering jurisdictions with CWD-positive animals can increase the likelihood of disease spread. Additional risk factors, such as the presence of scrapie in sheep populations that are sympatric with deer and elk (Greenlee and others, 2011), feeding of animal protein to cervids (Johnson, McKenzie, and others, 2011), baiting and feeding programs (Thompson and others, 2008), or other environmental factors also may be considered, although their roles in CWD epidemiology has not been clearly established.

The soil composition of a region may also play an important role in the occurrence and maintenance of CWD and other TSEs (Smith and others, 2011). Recently, it has been shown that certain soil types can chemically bind and increase infectivity of prion protein ( $\mathrm{PrP})$, creating the potential for the protein to be maintained at the soil surface for uptake by foraging animals (Johnson and others, 2006; Johnson and others, 2007; Polano and others, 2008; Imrie, 2009); however, the fate of prions may be highly dependent on source of deposition into the soil (for example, fluid or tissue; Saunders and others 2009). In addition, organic soil components (humic acids) appear to enhance the adsorption of PrP to clay minerals and show a great affinity for the protein as well; however, it is unclear whether the effect of the organic matter increases or decreases infectivity (Polano and others, 2008). The importance of soil in CWD epidemiology was reaffirmed by Walter and others (2011) who demonstrated an 8.9-percent increase in an individual's deer's odds of CWD infection with each 1-percent increase in soil clay content within its approximate home range in north-central Colorado. These results suggest that some regions may have a greater probability of maintaining and spreading CWD based solely on their geologic and chemical attributes. Thus, the soil characteristics within an animal's range represent a potentially important spatial risk factor for CWD occurrence and maintenance. 
The risk of disease amplification (increasing the number of infected animals) in a target population or location postexposure likely increases as cervid population densities increase and predation decreases. In north-central Colorado, the greater the area of a mule deer's (Odocoileus hemionus) approximate home range that contained wintering concentration areas of deer (high deer densities) the greater the odds of individual CWD infection (Farnsworth and others, 2006; Walter and others, 2011). The absence of predators or harvest potentially allows infected animals a longer period when they can transmit CWD to other animals by direct contact or indirectly through environmental reservoirs (Wild and others, 2011). Removal of infectious animals by harvest or other means likely reduces the rate of disease transmission and prevalence in free-ranging cervids (Gross and Miller, 2001; Schauber and Woolf, 2003; Wasserberg and others, 2009; Habib and others, 2011), deposition of infectious prions into the environment, and the rate of disease spread. However, if infection rates are high and sustained, even intense selective predation of infected individuals may not reduce the spread and persistence of CWD (Miller and others, 2008), possibly due to the effects of indirect transmission on the disease process (Almberg and others, 2011). Baiting or feeding, which artificially increases concentrations of animals, may increase the chance of disease spread through direct contact among animals or indirect contact with environmental contamination (Thompson and others, 2008; Mathiason and others, 2009; Tamguney and others, 2009; Haley and others, 2011). Thus, variation in density of deer or infected deer across the landscape is another important spatial risk factor to consider when conducting disease surveillance or monitoring (Joly and others, 2009).

Habitat attributes and landscape features that influence animal distribution, movements, and behavior may be other factors affecting the risk of disease amplification. For example, laboratory experiments have demonstrated that some extracts from certain lichen species produce an enzyme (serine protease) that degrades $\operatorname{PrP}^{\mathrm{TSE}}$, potentially allowing these organisms to reduce TSE infectivity in the environment (Johnson, Bennett, and others, 2011). Although the importance of these findings outside the laboratory has not been ascertained, the presence and amount of certain species of lichens in the environment may influence the amount of infectious prion material available for uptake by uninfected individuals. This could impact the rate of CWD infection. The use of landscape genetics has demonstrated that landscape features that inhibit or promote deer movements (Blanchong and others, 2008; Kelly and others, 2010; Cullingham, Merrill, and others, 2011) and distance from the outbreak origin (Joly and others, 2006; Osnas and others, 2009; Robinson, 2011) can affect CWD spread. CWD prevalence in an area is correlated with the amount of deer habitat available (Joly and others, 2006). In addition, Habib and others (2011) conducted computer simulations suggesting that landscape composition, habitat characteristics, and resource selection by deer may affect contact rates and associated risk of infection. These simulation results are supported by findings of Silbernagel and others (2011) who investigated interactions of mule deer in the Saskatchewan CWD endemic zone. They discovered that habitat type played an important role in the proximity and potential contacts of deer collared with global positioning system (GPS) devices to one another, which suggests that habitat may be an important factor in CWD transmission. Despite a limited understanding of the effects of specific habitat attributes and ecological characteristics, it is clear they can play a significant role in disease dynamics and spread and should be considered as spatial risk factors whenever possible.

Anthropogenic activities, management policies, and land use patterns within an area may also be important considerations when designing CWD surveillance or monitoring strategies. The amount of private land within a deer's approximate home range has been shown to influence CWD infection rates in Colorado (Farnsworth and others, 2006; Walter and others, 2011). Although no causal mechanism has been established, it is probable that private lands may act as refugia from harvest pressure (Vieira and others, 2003), and private lands may often occur on more moist and productive soils, providing better production and habitat that may be selected for by cervids. In addition, these soils may be composed of soil types that may increase the infectivity of the prions as previously described. These conditions can concentrate animals, increase density, and affect the age-structure of local populations, which consequently affects disease dynamics. Management policies at multiple scales can also affect infection risk. For example, limited or no harvest or predation of deer in urban areas, compared to undeveloped areas, may promote higher densities of deer and prevent the removal of infected individuals (Farnsworth and others, 2005), thus increasing disease prevalence (Wasserberg and others, 2009). Likewise, spatially and temporally varying harvest regulations and management strategies across a jurisdiction affects cervid densities and population structure ( age and sex ratios), and may create spatial variability in CWD infection risk (Gross and Miller, 2001; Wasserberg and others, 2009; Bergman and others, 2011; Sharp and Pastor, 2011).

Identification and evaluation of CWD spatial risk factors helps to focus limited resources on locations or target populations with a greater likelihood of being infected. Presently, the ability to quantify the importance of spatial risk factors remains limited, and determination of their importance for any specific area must rely on available knowledge and the judgment and experience of wildlife and disease experts. Understanding the distribution, movement, social behavior, population characteristics, and dynamics of affected deer and elk populations, as well as the anthropogenic forces acting on these populations, is helpful, if not essential, to fully evaluate the spatial risk factors for CWD in free-ranging populations. 
Enhanced Surveillance Strategies for Detecting and Monitoring Chronic Wasting Disease in Free-Ranging Cervids

\section{Demographic Risk Factors}

Demographic risk factors are specific attributes of groups of individuals within a target population or the target population itself that are associated with prevalence of CWD. Several demographic risk factors have been linked to CWD prevalence including sex, age, density, social interactions, genetics, mortality factors, and clinical signs of CWD. Clinical signs include loss of fear of humans, ataxia, marked weakness, inability to stand, dehydration, rough dull hair coat, excessive salivation, drooping of the head and ears, and severe emaciation (Williams and Young, 1980; Spraker and others, 1997).

As research, surveillance, and monitoring efforts have continued following the discovery of CWD in free-ranging cervids, evidence has mounted demonstrating the validity of these attributes as factors influencing disease prevalence. For example, prevalence rates across sex and age classes of hunter-killed animals, culled individuals, and research activities demonstrated heterogeneity in prevalence across these various classes (Miller and Conner, 2005; Farnsworth and others, 2006; Osnas and others, 2009; Heisey and others, 2010; Walsh and Miller, 2010). Because the probability of CWD infection increases with time of exposure (age), older deer have higher prevalence of disease than fawns or yearlings. In Colorado and Wisconsin, adult male deer have a twofold higher prevalence than females, and within each sex class prevalence was considerably higher in adult animals compared to fawns or yearlings (Miller and Conner, 2005; Grear and others, 2006; Osnas and others, 2009; Heisey and others, 2010).

Different prevalence rates have also been reported in individuals succumbing to various mortality factors. Krumm and others (2005) reported that vehicle-killed mule deer had higher prevalence $(0.146)$ than hunter-harvested deer $(0.075)$ collected from the same vicinity. In addition, they found CWD-infected mule deer in later stages of the disease (with spongiform encephalopathy) were more frequently killed by vehicles compared to hunter-killed samples. However, CWD infected white-tailed deer in Wisconsin were not more vulnerable to harvest than uninfected deer (Grear and others, 2006). Research has also demonstrated an increased prevalence in predator-killed mule deer (Miller and others, 2008; Krumm and others, 2010). Thus, CWD-infected individuals can be more susceptible to some mortality factors than uninfected animals.

It has also been recognized that targeted surveillance of individuals demonstrating clinical signs consistent with CWD infection results an increased probability of detecting CWD. In one study, 66 percent and 43 percent of submitted deer and elk exhibiting clinical signs of CWD, respectively, were diagnosed as CWD-positive compared to 5 percent and 0.5 percent of randomly sampled deer and elk submitted for testing (Miller and others, 2000). Therefore, exhibition of clinical signs of CWD is a clearly an attribute that increases an individual's likelihood of having CWD and is a demographic risk factor that can be exploited to increase surveillance efficiency.
Targeted surveillance and investigation of clinical animals also has the added benefit of detection of other important wildlife diseases with similar clinical signs (Miller and others, 2000; Samuel and others, 2003).

Other demographic risk factors are less well understood. For example, there is evidence that genetics plays a role in individual susceptibility and rate of disease progression. Similar to other TSEs, polymorphisms of the prion protein gene (PRNP) may moderate individual susceptibility to and progression of CWD infection of elk, mule, and white-tailed deer (O'Rourke and others, 2004; Spraker and others, 2004; Jewell and others, 2005; Fox and others, 2006; Hamir and others, 2006; Goldmann, 2008; Keane and others, 2008; Perucchini and others, 2008). Therefore, it appears that certain individuals are innately at higher risk of CWD infection based solely on their PRNP genotype. For example, Wisconsin white-tailed deer with the PRNP genotype G96G have approximately four times higher rate of infection and 8 months shorter survival after infection compared to G96S deer (Robinson and others, 2012). However, unlike other TSEs, there is no evidence that any of the PRNP genotypes in wild cervids are immune to CWD infection.

Genetic relatedness, a measure of the proportion of genes shared between two individuals, has also been demonstrated to be important in CWD disease dynamics, undoubtedly owing to the fact that related individuals are likely to have more frequent contact or share similar risk factors (Blanchong and others, 2008; Ernest and others, 2010; Grear and others, 2010; Kelly and others, 2010; Cullingham, Nakada, and others, 2011). Genetic relatedness also affects social structure and associated interactions of cervids, thereby affecting an individual's disease risk (Altizer and others, 2003). Farnsworth and others (2006) determined that patterns of CWD prevalence were best explained at the scale of an individual's home range, suggesting that local interactions within matrilineal groups influences CWD dynamics. Likewise, several studies have found that CWD-infected female deer had increased genetic relatedness compared to sympatric non-infected deer (Blanchong and others, 2008; Grear and others, 2010; Cullingham, Nakada, and others, 2011). Schauber and others (2007) demonstrated that contact rates between white-tailed deer within social groups were significantly higher than contact rates of individuals between social groups, providing insight into a possible mechanism for these findings. In a further refinement, Silbernagel and others (2011) found direct and indirect contact varied by season and habitat characteristics. These studies suggest female relatives of an infected female are at higher risk of either direct or indirect transmission owing to their philopatric behaviors and formation of matriarchal groups (Altizer and others, 2003; Conner and Miller, 2004; Grear and others, 2006; Joly and others, 2006; Blanchong and others, 2008; Grear and others, 2010; Kelly and others, 2010; Cullingham, Nakada, and others, 2011; Cullingham, Merrill, and others, 2011). Thus, genetic relatedness and associated social interactions are important risk factors. However, despite the clear 
importance of genetic relatedness in determining an individual's CWD-infection risk, its contribution to prevalence of CWD in wild cervid populations is not yet clearly understood.

Finally, high population density, which also can be considered a spatial risk factor, is generally believed to create increased risk of disease transmission through higher direct or indirect contact rates (Swinton and others, 2001; Ramsey and others, 2002). This is the basis for population reduction strategies used by many wildlife management agencies for CWD management in free-ranging cervids (Williams and others, 2002; Joly and others, 2003; Williams, 2005; Joly and others, 2006). The actual transmission route of CWD is not known, however, experimental evidence from captive cervids suggests that CWD infection occurs via horizontal transmission through both direct and indirect contact between susceptible and infected individuals (Miller and Williams, 2003; Williams and Miller, 2003), and both kinds of contact can be influenced by density. Experimental inoculation with blood, urine, feces, and saliva from CWD-infected individuals has been shown to provide viable routes of transmission, suggesting direct contact with any of these infectious materials could act as a route of infection (Miller and others, 2004; Mathiason and others, 2006; Miller and others, 2006; Trifilo and others, 2007; Safar and others, 2008; Haley and others, 2009; Mathiason and others, 2009; Tamguney and others, 2009; Haley and others, 2011). Indirect contact may play an important role in transmission dynamics via environmental contamination, because the CWD agent can persist in contaminated systems for 2 or more years (Miller and others, 2004), and if CWD is similar to scrapie, it may persist for 15 or more years (Georgsson and others, 2006). For captive cervids, the most likely route of exposure is orally through foraging activities in the immediate vicinity of fresh and decomposed carcasses or ingestion of fresh and residual excreta from infected individuals (Miller and others, 2004; Trifilo and others, 2007; Safar and others, 2008; Mathiason and others, 2009). However, the relative importance of direct and indirect transmission of CWD in wild cervids has not been determined. As previously mentioned, certain soil types can also increase oral infectivity of TSEs, which may allow environmental contamination to be problematic even in the presence of relatively low doses of the infective agent (Johnson and others, 2007). Thus, it is likely density of infected cervids can contribute to increased indirect contact rate between susceptible and infected individuals or contact with an environmental reservoir. However, it is unclear to what the extent density influences these processes.

Although the risk associated with several demographic factors is not completely understood, current knowledge is adequate to allow for incorporation of several factors into surveillance activities. By using information on differential risk factors in surveillance and monitoring activities is critical, because it can increase efficiency of disease detection and allow wildlife management agencies to evolve and improve surveillance and monitoring efforts. In addition, as our understanding of the effects of other demographic parameters on CWD prevalence increase, this information can be incorporated to further streamline disease-management programs.

\section{Conclusion}

We have presented a wide range of risk factors that may be considered when designing surveillance or monitoring programs. Although this is not an exhaustive list and undoubtedly other factors not yet described will come to light, the risk factors and associated references detailed herein provide a useful starting point. Many jurisdictions currently collect information on many of these factors, especially for harvested cervids. Other data related to spatial risk factors may be readily available within Geographic Information Systems (GIS). Thus, the use of the risk factors in surveillance and monitoring activities is a natural progression in continued improvement of these activities.

For jurisdictions lacking quantitative information on various risk factors, we recommend collaborating with similar entities that have available information and utilizing their data to incorporate risk factors into disease-management activities. In addition, even if information is limited or unavailable for some of the risk factors described, use of the information that is available for the remaining factors may provide gains in efficiency. However, we caution that, if possible, risk factors related to CWD infection should also consider potential differences in species of interest, differences in harvest strategies among areas, if CWD is in an early or advanced stage of epizootic (low vs. high prevalence), and how different cervid ecology and habitat features might affect estimate risk factors. At the current time, little is known about how many of these features might affect CWD prevalence and risk of infection.

Evaluation of risk factors helps to focus resources on locations or target populations with a greater likelihood of being infected and increases the efficiency of surveillance and monitoring efforts. Agencies charged with managing this disease should continue to look for efficient ways to conduct their activities. Identifying, understanding the importance of, and incorporating risk factors into surveillance and monitoring activities are potential means of meeting this need for increased efficiency. 


\section{Literature Cited}

Almberg, E.S.; Cross, P.C.; Johnson, C.J.; Heisey, D.M.; and Richards, B.J., 2011, Modeling routes of chronic wasting disease transmission: Environmental prion persistence promotes deer population decline and extinction: PLoS ONE, v. 6 , no. 5 .

Altizer, S.; Nunn, C.L.; Thrall, P.H.; Gittleman, J.L.; Antonovics, J.; Cunningham, A.A.; Dobson, A.P.; Ezenwa, V.; Jones, K.E.; Pedersen, A.B.; Poss, M.; and Pulliam, J.R.C., 2003, Social organization and parasite risk in mammals: Integrating theory and empirical studies. Annual Review of Ecology, Evolution, and Systematics, v. 34, p. 517-547.

Bergman, E.J.; Watkins, B.E.; Bishop, C.J.; Lukacs, P.M.; and Lloyd, M., 2011, Biological and socio-economic effects of statewide limitation of deer licenses in Colorado: Journal of Wildlife Management, v. 75, p. 1443-1452.

Blanchong, J.A.; Samuel, M.D.; Scribner, K.T.; Weckworth, B.V.; Langenberg, J.A.; and Filcek, K.B., 2008, Landscape genetics and the spatial distribution of chronic wasting disease: Biology Letters, v. 4, p. 130-133.

Conner, M.M., and Miller, M.W., 2004, Movement patterns and spatial epidemiology of a prion disease in mule deer population units: Ecological Applications, v. 14, p. 18701881.

Cullingham, C.I.; Merrill, E.H.; Pybus, M.J.; Bollinger, T.K.; Wilson, G.A.; and Coltman, D.W., 2011, Broad and finescale genetic analysis of white-tailed deer populations: Estimating the relative risk of chronic wasting disease spread: Evolutionary Applications, v. 4, p. 116-131.

Cullingham, C.I.; Nakada, S.M.; Merrill, E.H.; Bollinger, T.K.; Pybus, M.J.; and Coltman, D.W., 2011, Multiscale population genetic analysis of mule deer (Odocoileus hemionus hemionus) in western Canada sheds new light on the spread of chronic wasting disease: Canadian Journal of ZoologyRevue Canadienne De Zoologie, v. 89, p. 134-147.

Doherr, M.G.; Heim, D.; Fatzer, R.; Cohen, C.H.; Vandevelde, M.; and Zurbriggen, A., 2001, Targeted screening of high-risk cattle populations for BSE to augment mandatory reporting of clinical suspects: Preventive Veterinary Medicine, v. 51, p. 3-16.

Ernest, H.B.; Hoar, B.R.; Well, J.A.; and O’Rourke, K.I., 2010, Molecular genealogy tools for white-tailed deer with chronic wasting disease: Canadian Journal of Veterinary Research, v. 74, p. 153-156.

Farnsworth, M.L.; Hoeting, J.A.; Hobbs, N.T.; and Miller, M.W., 2006, Linking chronic wasting disease to mule deer movement scales: A hierarchical Bayesian approach: Ecological Applications, v. 16, p. 1026-1036.
Farnsworth, M.L.; Wolfe, L.L.; Hobbs, N.T.; Burnham, K.P.; Williams, E.S.; Theobald, D.M.; Conner, M.M.; and Miller, M.W., 2005, Human land use influences chronic wasting disease prevalence in mule deer: Ecological Applications, v. 15 , p. 119-126.

Fox, K.A.; Jewell, J.E.; Williams, E.S.; and Miller, M.W., 2006, Patterns of Prp(CWD) accumulation during the course of chronic wasting disease infection in orally inoculated mule deer (Odocoileus hemionus): Journal of General Virology, v. 87, p. 3451-3461.

Georgsson, G.; Sigurdarson, S.; and Brown, P., 2006, Infectious agent for sheep scrapie may persist in the environment for at least 16 years: Journal of General Virology, v. 87, p. $3737-3740$.

Goldmann, W., 2008, PrP genetics in ruminant transmissible spongiform encephalopathies: Veterinary Research, v. 39, no. 4.

Grear, D.A.; Samuel, M.D.; Langenberg, J.A.; and Keane, D., 2006, Demographic patterns and harvest vulnerability of chronic wasting disease infected white-tailed deer in Wisconsin: Journal of Wildlife Management, v. 70, p. 546-533.

Grear, D.A.; Samuel, M.D.; Scribner, K.T.; Weckworth, B.V.; and Langenberg, J.A., 2010, Influence of genetic relatedness and spatial proximity on chronic wasting disease infection among female white-tailed deer:Journal of Applied Ecology, v. 47, p. $532-540$.

Greenlee, J.J.; Smith, J.D.; and Kunkle, R.A., 2011, Whitetailed deer are susceptible to the agent of sheep scrapie by intracerebral inoculation: Veterinary Research, v. 42, article 107.

Gross, J.E., and Miller, M.W., 2001, Chronic wasting disease in mule deer: Disease dynamics and control: The Journal of Wildlife Management, v. 65, p. 205-215.

Habib, T.J.; Merrill, E.H.; Pybus, M.J.; and Coltman, D.W., 2011, Modelling landscape effects on density-contact rate relationships of deer in eastern Alberta: Implications for chronic wasting disease: Ecological Modelling, v. 222, p. 2722-2732.

Haley, N.J.; Mathiason, C.K.; Carver, S.; Zabel, M.; Telling, G.C.; and Hoover, E.A., 2011, Detection of chronic wasting disease prions in salivary, urinary, and intestinal tissues of deer: Potential mechanisms of prion shedding and transmission: Journal of Virology, v. 85, p. 6309-6318.

Haley, N.J.; Seelig, D.M.; Zabel, M.D.; Telling, G.C.; and Hoover, E.A., 2009, Detection of CWD prions in urine and saliva of deer by transgenic mouse bioassay: PLoS ONE, no. 4. 
Hamir, A.N.; Gidlewski, T.; Spraker, T.R.; Miller, J.M.; Creekmore, L.; Crocheck, M.; Cline, T.; and O’Rourke, K.I., 2006, Preliminary observations of genetic susceptibility of elk (Cervus elaphus nelsoni) to chronic wasting disease by experimental oral inoculation: Journal of Veterinary Diagnostic Investigation, no. 18, p. 110-114.

Heisey, D.M.; Osnas, E.E.; Cross, P.C.; Joly, D.O.; Langenberg, J.A.; and Miller, M.W., 2010, Linking process to pattern: Estimating spatiotemporal dynamics of a wildlife epidemic from cross-sectional data: Ecological Monographs, v. 80, p. 221-240.

Imrie, C.E., 2009, Spatial correlation between the prevalence of transmissible spongiform diseases and British soil geochemistry: Evironmental Geochemistry and Health, v. 31, p. 133-145.

Jewell, J.E.; Conner, M.M.; Wolfe, L.L.; Miller, M.W.; and Williams, E.S., 2005, Low frequency of PrP genotype 225SF among free-ranging mule deer (Odocoileus hemionus) with chronic wasting disease: Journal of General Virology, v. 86, p. 2127-2134.

Johnson, C.J.; Bennett, J.P.; Biro, S.M.; Duque-Velasquez, J.C.; Rodriguez, C.M.; Bessen, R.A.; and Rocke, T.E., 2011, Degradation of the disease-associated prion protein by a serine protease from lichens: PLoS ONE, v. 6.

Johnson, C.J.; McKenzie, D.; Pedersen, J.A.; and Aiken, J.M., 2011, Meat and bone meal and mineral feed additives may increase the risk of oral prion disease transmission: Journal of Toxicology and Environmental Health, Part A, v. 74, p. 161-166.

Johnson, C.J.; Pedersen, J.A.; Chappell, R.J.; McKenzie, D.; and Aiken, J.M., 2007, Oral transmissibility of prion disease is enhanced by binding to soil particles: PLOS Pathogens, v. 3, p. 874-881.

Johnson, C.J.; Phillips, K.E.; Schramm, P.T.; McKenzie, D.; Aiken, J.M.; and Pedersen, J.A., 2006, Prions adhere to soil minerals and remain infectious: PLOS Pathogens, v. 2, p. 296-302.

Joly, D.O.; Ribic, C.A.; Langenberg, J.A.; Beheler, K.; Batha, C.A.; Dhuey, B.J.; Rolley, R.E.; Bartelt, G.; Van Deelen, T.R.; and Samuel, M.D., 2003, Chronic wasting disease in free-ranging Wisconsin white-tailed deer: Emerging Infectious Diseases, v. 9, p. 599-601.

Joly, D.O.; Samuel, M.D.; Langenberg, J.A.; Blanchong, J.A.; Batha, C.A.; Rolley, R.E.; Keane, D.P.; and Ribic, C.A., 2006, Spatial epidemiology of chronic wasting disease in Wisconsin white-tailed deer: Journal of Wildlife Diseases, v. 42 , p. $578-588$.
Joly, D.O.; Samuel, M.D.; Langenberg, J.A.; Rolley, R.E.; and Keane, D.P., 2009, Surveillance to detect chronic wasting disease in white-tailed deer in Wisconsin: Journal of Wildlife Diseases, v. 45, p. 989-997.

Keane, D.P.; Barr, D.J.; Boschler, P.N.; Hall, S.M.; Gidlewski, T.; O’Rourke, K.I.; Spraker, T.R.; and Samuel, M.D., 2008, Chronic wasting disease in a Wisconsin white-tailed deer farm: Journal of Veterinary Diagnostic Investigation, v. 20, p. 698-703.

Kelly, A.C.; Mateus-Pinilla, N.E.; Douglas, M.; Brown, W.; Ruiz, M.O.; Killefer, J.; Shelton, P.; Beissel, T.; and Novakofski, J., 2010, Utilizing disease surveillance to examine gene flow and dispersal in white-tailed deer: Journal of Applied Ecology, v. 47, p. 1189-1198.

Krumm, C.E.; Conner, M.M.; and Miller, M.W., 2005, Relative vulnerability of chronic wasting disease infected mule deer to vehicle collisions: Journal of Wildlife Diseases, v. 41, p. 503-511.

Krumm, C.E.; Conner, M.M.; Hobbs, N.T.; Hunter, D.O.; and Miller, M.W., 2010, Mountain lions prey selectively on prion-infected mule deer: Biology Letters, v. 6, p. 209-211.

Mathiason, C.K.; Hays, S.A.; Powers, J.; Hayes-Klug, J.; Langenberg, J.; Dahmes, S.J.; Osborn, D.A.; Miller, K.V.; Warren, R.J.; Mason, G.L.; and Hoover, E.A., 2009, Infectious prions in pre-clinical deer and transmission of chronic wasting disease solely by environmental exposure: PLoS ONE, v. 4.

Mathiason, C.K.; Powers, J.G.; Dahmes, S.J.; Osborn, D.A.; Miller, K.V.; Warren, R.J.; Mason, G.L.; Hays, S.A.; Hayes-Klug, J.; Seelig, D.M.; Wild, M.A.; Wolfe, L.L.; Spraker, T.R.; Miller, M.W.; Sigurdson, C.J.; Telling, G.C.; and Hoover, E.A., 2006, Infectious prions in the saliva and blood of deer with chronic wasting disease: Science, v. 314, p. 133-136.

Miller, M.W., and Conner, M.M., 2005, Epidemiology of chronic wasting disease in free-ranging mule deer: Spatial, temporal, and demographic influences on observed prevalence patterns: Journal of Wildlife Diseases, v. 41, p. $275-290$.

Miller, M.W.; Hobbs, N.T.; and Tavener, S.J., 2006, Dynamics of prion disease transmission in mule deer: Ecological Applications, v. 16, p. 2208-2214.

Miller, M.W.; Swanson, H.M.; Wolfe, L.L.; Quartarone, F.G.; Huwer, S.L.; Southwick, C.H.; and Lukacs, P.M., 2008, Lions and prions and deer demise: PLoS ONE, v. 3, no. 12, e4019, http://dx.doi.org/doi:10.1371/journal.pone.0004019. 
Miller, M.W., and Williams E.S., 2003, Horizontal prion transmission in mule deer: Nature, v. 425, p. 35-36.

Miller, M.W.; Williams, E.S.; Hobbs, N.T.; and Wolfe, L.L., 2004, Environmental sources of prion transmission in mule deer: Emerging Infectious Diseases, v. 10, p. 1003-1006.

Miller, M.W.; Williams, E.S.; McCarty, C.W.; Spraker, T.R.; Kreeger, T.J.; Larsen, C.T.; and Thorne, E.T., 2000, Epizootiology of chronic wasting disease in free-ranging cervids in Colorado and Wyoming: Journal of Wildlife Diseases, v. 36, p. 676-690.

O’Rourke, K.I.; Spraker, T.R.; Hamburg, L.K.; Besser, T.E.; Brayton, K.A.; and Knowles, D.P., 2004, Polymorphisms in the prion precursor functional gene but not the pseudogene are associated with susceptibility to chronic wasting disease in white-tailed deer: Journal of General Virology, v. 85, p. 1339-1346.

Osnas, E.E.; Heisey, D.M.; Rolley, R.E.; and Samuel, M.D., 2009, Spatial and temporal patterns of chronic wasting disease: Fine-scale mapping of a wildlife epidemic in Wisconsin: Ecological Applications, v. 19, p. 1311-1322.

Perucchini, M.; Griffin, K.; Miller, M.W.; and Goldmann, W., 2008, PrP genotypes of free-ranging wapiti (Cervus elaphus nelsoni) with chronic wasting disease: Journal of General Virology, v. 89, p. 1324-1328.

Polano, M.; Anslemi, C.; Leita, L.; Negro, A.; and Nobili, M.D., 2008, Organic polyanions act as complexants of prion protein in soil: Biochemical and Biophysical Research Communications, v. 267, p. 323-329.

Ramsey, D.; Spencer, N.; Caley, P.; Efford, M.; Hansen, K.; Lam, M.; and Cooper, D., 2002, The effects of reducing population density on contact rates between brushtail possums: Implications for transmission of bovine tuberculosis: Journal of Applied Ecology, v. 39, p. 806-818.

Robinson, S.J., 2011, Landscape genetics meets landscape epidemiology in the chronic wasting disease zone of the Midwestern USA: How the landscape shapes connectivity and disease spread among populations of white-tailed deer: Madison, Wis., University of Wisconsin, Ph.D. dissertation.

Robinson, S.J.; Samuel, M.D.; Johnson, C.J.; Adams, M.; and McKenzie, D.I., in press, Emerging prion disease drives host selection in a wildlife population: Ecological Applications.

Safar, J.G.; Lessard, P.; Tamgueney, G.; Freyman, Y.; Deering, C.; Letessier, F.; DeArmond, S.J.; and Prusiner, S.B., 2008, Transmission and detection of prions in feces: Journal of Infectious Diseases, v. 198, p. 81-89.
Samuel, M.D.; Joly, D.O.; Wild, M.A.; Wright, S.D.; Otis, D.L.; Werge, R.W.; and Miller, M.W., 2003, Surveillance strategies for detecting chronic wasting disease in free-ranging deer and elk: Results of a CWD surveillance workshop, 10-12 December 2002: U.S. Geological Survey National Wildlife Health Center, 40 p., accessed May 27, 2011, at http://www.nwhc.usgs.gov/publications/fact_sheets/pdfs/ cwd/CWD_Surveillance_Strategies.pdf.

Saunders, S.E.; Bartz, J.C.; and Bartelt-Hunt, S.L., 2009, Influence of prion strain on prion protein adsorption to soil in a competitive matrix: Environmental Science and Technology, v. 43, p. 5242-5248.

Schauber, E.M.; Storm, D.J.; and Nielsen, C.K., 2007, Effects of joint space use and group membership on contact rates among white-tailed deer: Journal of Wildlife Management, v. 71, p. 155-163.

Schauber, E.M., and Woolf, A., 2003, Chronic wasting disease in deer and elk: A critique of current models and their application: Wildlife Society Bulletin, v. 31, p. 610-616.

Sharp, A., and Pastor J., 2011, Stable limit cycles and the paradox of enrichment in a model of chronic wasting disease: Ecological Applications, v. 21, p. 1024-1030.

Shelton, P., and McDonald, P., 2011, Illinois chronic wasting disease: 2010-2011 surveillance management summary: Illinois Department of Natural Resources, 16 p., accessed Nov. 18, 2011, at http://dnr.state.il.us/cwd/.

Silbernagel, E.R.; Skelton, N.K.; Waldner, C.L.; and Bolliner, T.K., 2011, Interaction among deer in a chronic wasting disease endemic zone: Journal of Wildlife Management, v. 75 , p. 1453-1461.

Smith, C.B.; Booth, C.J.; and Pedersen, J.A., 2011, Fate of prions in soil: A review: Journal of Environmental Quality, v. 40, p. 449-461.

Spraker, T.R.; Balachandran, A.; Zhuang, D.; and O’Rourke, K.I., 2004, Variable patterns of distribution of Prp(CWD) in the obex and cranial lymphoid tissues of Rocky Mountain elk (Cervus elaphus nelsoni) with subclinical chronic wasting disease: Veterinary Record, v. 155, p. 295-302.

Spraker, T.R.; Miller, M.W.; Williams, E.S.; Getzy, D.M.; Adrian, W.J.; Schoonveld, G.G.; Spowart, R.A.; Orourke, K.I.; Miller, J.M.; and Merz, P.A., 1997, Spongiform encephalopathy in free-ranging mule deer (Odocoileus hemionus), white-tailed deer (Odocoileus virginianus) and Rocky Mountain elk (Cervus elaphus nelsoni) in northcentral Colorado: Journal of Wildlife Diseases, v. 33, p. 1-6. 
Stark, K.D.; Regula, G.; Hernandez, J.; Knopf, L.; Fuchs, K.; Morris, R.S.; and Davies, P., 2006, Concepts for risk-based surveillance in the field of veterinary medicine and veterinary public health: Review of current approaches: BMC Health Services Research, v. 6, p. 20.

Swinton, J.; Woolhouse, M.E.J.; Begon, M.E.; Dobson, A.P.; Ferroglio, E.; Grenfell, B.T.; Guberti, V.; Hails, R.S.; Heesterbeek, J.A.P.; Lavazza, A.; Roberts, M.G.; White, P.J.; and Wilson, K., 2001, Micorparasite transmission and persistence, in Hudson, P.J.; Rizzoli, A.; Grenfell, B.T.; Heesterbeek, H.; and Dobson, A.P. (eds.), The ecology of wildlife diseases: New York, Oxford University Press, p. $83-101$.

Tamguney, G.; Miller, M.W.; Wolfe, L.L.; Sirochman, T.M.; Glidden, D.V.; Palmer, C.; Lemus, A.; DeArmond, S.J.; and Prusiner, S.B., 2009, Asymptomatic deer excrete infectious prions in faeces: Nature, v. 461, p. 529-590.

Thompson, A.K.; Samuel, M.D.; and Deelen, T.R.V., 2008, Alternative feeding stategies and potential disease transmission in Wisconsin white-tailed deer: Journal of Wildlife Management, v. 72, p. 416-421.

Trifilo, M.J.; Ying, G.; Teng, C.; and Oldstone, M.B.A., 2007, Chronic wasting disease of deer and elk in transgenic mice: Oral transmission and pathobiology: Virology, v. 365, p. 136-143.

Vieira, M.E.P.; Conner, M.M.; White, G.C.; and Freddy, D.J., 2003, Effects of archery hunter numbers and opening dates on elk movement: Journal of Wildlife Management, v. 67, p. 717-728.

Walsh, D.P., and Miller, M.W., 2010, Designing a weighted surveillance system for chronic wasting disease in Colorado: Journal of Wildlife Diseases, v. 46, p. 118-135.

Walter, W.D.; Walsh, D.P.; Miller, M.W.; Winkelman, D.L.; and Farnsworth, M.L., 2011, Soil clay content underlies prion infection odds: Nature Communications, v. 2, article 200, http://dx.doi.org/10.1038/ncomms1203.
Wasserberg, G.; Onas, E.E.; Rolley, R.E.; and Samuel, M.D., 2009, Host culling as an adaptive management tool for chronic wasting disease-A modeling study: Journal of Applied Ecology, v. 46, p. 457-466.

Wild, M.A.; Hobbs, N.T.; Graham, M.S.; and Miller, M.W., 2011, The role of predation in disease control: A comparison of selective and nonselective removal on prion disease dynamcis in deer: Journal of Wildlife Diseases, v. 47, p. $78-93$.

Wilesmith, J.; Morris, R.; Stevenson, M.; Cannon, R.; Prattley, D.; and Benard. H., 2004, Development of a method for evaluation of national surveillance data and optimization of national surveillance strategies for bovine spongiform encephalopathy: Weybridge, United Kingdom. European Union TSE Community Reference Laboratory, $34 \mathrm{p}$.

Williams, E.S., 2005, Chronic wasting disease: Veterinary Pathology, v. 42, p. 530-549.

Williams, E.S., and Miller, M.W., 2003, Transmissible spongiform encephalopathies in non-domestic animals: Origin, transmission and risk factors: Revue Scientifique et Technique de l' Office International Des Epizooties, v. 22, p. 145-156.

Williams, E.S.; Miller, M.W.; Kreeger, T.J.; Kahn, R.H.; and Thorne, E.T., 2002, Chronic wasting disease of deer and elk: A review with recommendations for management: Journal of Wildlife Management. v. 66, p. 551-563.

Williams, E.S., and Young, S., 1980, Chronic wasting disease of captive mule deer: A spongiform encephalopathy: Journal of Wildlife Diseases, v. 16, p. 89-98.

Wyoming Game and Fish Department, 2010, Wyoming chronic wasting disease (CWD) percent positive deer by hunt area 1978-2010: accessed Nov. 28, 2011, at http://gf.state.wy.us/services/education/cwd/index.asp. 



\title{
Chapter 2 \\ Disease Surveillance: Incorporating Available Information to Enhance Disease-Detection Efforts
}

\author{
By Daniel P. Walsh, Ph.D.', and David L. Otis, Ph.D. ${ }^{2}$
}

\section{Introduction}

Disease surveillance is "an active, on-going, formal, and systematic process aimed at early detection of a specific disease or agent in a population, or early prediction of elevated risk of a population acquiring an infectious disease, with a prespecified action that would follow the detection of disease" (Thurmond, 2003). Thus, the main goal of any diseasesurveillance program is the ability to detect the presence of the disease as early as possible in hopes of being able to respond and minimize adverse impacts of the disease.

The heightened public awareness, governmental concern, and improved diagnosis of infectious animal and zoonotic diseases, as well as the increased movement of animals, including wildlife, nationally and internationally has created demand for effective disease-surveillance programs (Stark and others, 2006). Despite this demand, the amount of resources available for supporting these surveillance programs is finite, often with decreasing commitment over time as public concern wanes and other disease issues emerge. Therefore, the need for efficient programs is paramount if governmental agencies hope to meet their mandates to conduct disease surveillance.

One method of improving efficiency of disease discovery is to incorporate auxiliary information about specific segments of the target population that can be used to stratify these groups spatially and/or demographically, based on varying prevalence rates or heterogeneous disease risk that has been elucidated in epidemiological investigations where the disease occurs. This auxiliary information can be any covariate that enhances the ability to detect the disease of interest by focusing surveillance efforts on areas and individuals with the highest probability of being infected or, in other words, those at the greatest disease risk (Thurmond, 2003). This approach has been called "proportional risk sampling," "risk-based sampling," "targeted sampling," and herein "weighted sampling" (Doherr and others, 2001; Hadorn and others, 2002; Thurmond, 2003; Stark and others, 2006; Walsh and Miller, 2010).
This technique has been most widely documented and successfully used in bovine spongiform encephalopathy (BSE) surveillance in Europe and has been used with other animal diseases as well (Doherr and others, 2001; Hadorn and others, 2002; Wilesmith and others, 2004; Bohning and Greiner, 2006; Walsh and Miller, 2010).

In the context of surveillance for chronic wasting disease (CWD), this weighted surveillance system has potential to greatly increase the disease discovery efficiency of current efforts. As described in the previous chapter, several spatial and demographic risk factors can be used to inform this system and provide the necessary covariate information to focus sampling efforts on specific regions and groups, which enhances early detection of CWD. Thus, the samples entering the surveillance stream from these various groups are weighted according to their probability of being infected or at risk. By utilizing information by the weights, the number of samples required for testing is decreased without a resulting loss of statistical confidence in the findings of the surveillance system. The savings from this strategy can be significant and increase the overall effectiveness of CWD surveillance.

In the following pages, we describe how to design a CWD weighted surveillance system, including the sampling aspects: spatial and demographic, the incorporation of various risk factors, the calculation of weights, the practical implementation of the system, and the statistical theory upon which the system is based. We hope to provide a framework that interested individuals can use to design the most effective and efficient CWD surveillance system possible for their jurisdiction, given their current state of knowledge. We assume the reader is familiar with Samuel and others (2003) and the general principles, philosophies, and practicalities of CWD surveillance described therein and do not attempt to reiterate that information here. Rather, we focus solely on describing weighted surveillance systems and their utility when attempting to detect CWD on the landscape in a costeffective manner. We also discuss questions of temporal scale

${ }^{1}$ U.S. Geological Survey, National Wildlife Health Center.

${ }^{2}$ U.S. Geological Survey, Iowa Cooperative Fish and Wildlife Research Unit. 
of surveillance programs and determining when surveillance efforts have reached an adequate level and can be discontinued. It is important to emphasize that the techniques described in this chapter are specific to efforts to detect CWD where it is not yet known to occur on the landscape, and it is not intended to address disease investigations in regions where CWD is already known to be present (see Chapter 3).

\section{Weighted Surveillance System}

A disease-surveillance program should be designed with the goal to produce statistically reliable inferences about the expected probability of disease detection given specific assumptions about the relevant sampling and biological processes. Design-based survey sampling methodology provides a well-developed set of principles and protocols for choosing sampling units in such a way as to produce valid statistical inferences from the data. Practical and efficient sampling designs for detection of rare events (for example, disease) in free-ranging wildlife populations are notoriously difficult to implement (Thompson 2004), and statistical compromises are to be expected as a result. Our objective is, therefore, to suggest reasonable sampling protocols that can be expected to generate the same levels of CWD detection probability as traditional disease-sampling approaches with fewer samples and, therefore, fewer resources.

In any surveillance program, multiple and interactive economic, social, and biological factors affect choice of the sampling design. We cannot attempt to enumerate all the possibilities, but our intention in this brief discussion and presentation of examples is to illustrate that there is a rich collection of techniques for construction of sampling designs and that knowledge of risk factors, biology, and disease epidemiology can be formally used to determine design choices.

For our purposes, it is useful to think about any proposed sampling design as consisting of two general nested stages. In the first stage, we are concerned with the choice of explicit geographical units within which a sample of individual deer will be chosen for CWD testing. We refer to these selected geographical units as sampling units (SUs). The second sampling stage is concerned with how the individuals are chosen within a SU. As we shall see, sampling protocols for both stages can have important consequences for the statistical performance of the surveillance program, depending on the spatial distribution of the disease in the population.

For convenience, we define the target population as the population of individual deer within a State, although we note that methods described herein could be applied to any animal population in any region without loss of generality. The conceptual sampling frame is hierarchically structured into SUs and population elements (individual deer) within SUs. Sampling units could be townships, counties, game management units, or some other bureaucratic system of geographical boundaries determined by political mandates, cost efficiency, or biological factors.
In probability-based sampling designs, specific welldefined rules are invoked to select SUs and their population elements for measurement from the sampling frame. A required condition is that all elements have a controlled and non-zero probability of being selected. The selection rules and sampling frame allow us to calculate the probability that a population element is included in the sample and to derive estimators appropriate for a specific sample design. Random choice of SUs and population elements provides the basis for valid statistical inference to the population of interest.

Admittedly, it is often impractical or cost-prohibitive to implement a completely probability-based surveillance design. In particular, wildlife cannot be selected with controlled probability within an SU. However, we believe it is often possible to invoke probability sampling techniques (for example, stratification, cluster sampling, sampling with probability proportional to a risk factor) for at least some component of the sampling process, particularly in selection of SUs, which may reduce the impact of bias generated by convenience sampling or other non-probability-based sampling protocols.

\section{Probabilistic Sampling Designs for Selection of Sampling Units-Clustering and Stratification}

We will begin by examining sample designs for the first stage of sampling: selection of SUs. Survey sampling methodology provides two basic tools, clustering and stratification, for imposing additional control over the spatial distribution of the sample. When a clustering approach is taken, the motivation is usually cost efficiency. In our situation, reduced cost can be expected, because the clustering design will result in a more spatially concentrated selection of deer for testing. Use of multistage cluster sampling in a CWD surveillance program would involve imposing a hierarchical structure of groups of geographically adjacent SUs into clusters. For example, let us define a SU as a section (640 acres) of land. Rather than selecting a completely random sample of sections from the entire State, we first group SUs into townships (36 sections), and then randomly select a sample of townships (clusters). We next select a random subsample of sections (SUs) within each selected township and collect a sample of deer for measurement from these sections. This design is similar to a threestage cluster design, with townships as the first stage, sections as the second stage, and individual deer as the third stage; however we have no control over probability of deer selection.

A cluster sampling design can be used in conjunction with stratification, which is the second standard survey sampling tool for controlling spatial distribution of SUs. Motivation for stratification often derives from a desire to ensure more exhaustive spatial coverage of the target population than may occur by chance in completely random sampling of the entire population. In stratified sampling designs, the population is divided into mutually exclusive and exhaustive strata, and samples are selected from every stratum. For example, instead of selecting townships at random from the entire State, we might first define counties as strata and then proceed with 
the previously described cluster sampling approach in each county. As another example, instead of using political boundaries, we might use biologically based criteria such as major ecoregions within a State to create strata boundaries. Stratification can also be effective in increasing precision of estimates if the response of interest (disease prevalence) tends to be more homogeneous within strata than in the population as a whole. However, for surveillance sampling of a rare event in a spatially heterogeneous landscape, development of a practical stratification scheme that could improve statistical efficiency is unlikely. We believe it is preferable to define strata with the objective of increased control of spatial coverage of SUs, and then use the statistical technique of unequal probability sampling within strata to improve statistical efficiency.

\section{Unequal Probability Sampling and Risk Factors}

Survey sampling techniques can be applied that lead to probability-based over-sampling (sampling at a higher rate) of more informative SUs in a multistage cluster design. This is a form of unequal probability sampling called sampling with "probability proportional to size" (PPS). Size is a generic measure of the importance of some characteristic of an SU with respect to information on disease prevalence, and represents a formal method of more intensive sampling of more interesting units. In addition, if the size measure is directly related to the probability that the disease is present in the sample unit, then PPS leads to higher precision of the estimates (Lohr, 1999). For CWD surveillance programs, we strongly recommend construction of unequal probability sampling schemes based on knowledge of disease risk factors. If available, some factors, for example, soil clay content, number of captive cervid facilities, deer density, could be used directly in calculation of selection probabilities. Other indirect factors, such as an assumption about the spatial distribution of the disease- random or highly clustered - could also influence the choice of scale for sampling units or boundary definitions for strata.

As an illustration, suppose we want to randomly select a number (n) townships for sampling deer within a county comprised of $\mathrm{N}$ townships. If we select them completely at random or systematically, then each township has an equal probability of being chosen. However, suppose we want to give preference to townships with more woodland habitat, which we assume is highly correlated with deer density, which in turn may be correlated with increased probability of disease transmission. By using a geographic information system (GIS) coverage of the county, we can calculate the number of woodland acres in each township, and scale our probabilities of selection to be proportional to woodland acres. Now, although all townships still have a positive probability of being sampled, a township A with X hectares of woodland has double the chance of being sampled compared to a township B with only $\mathrm{X} / 2$ woodland hectares.

Unequal probabilities of selection could also be applied at any stage of the design. Consider a few examples:
1. Townships within counties are selected with probability proportional to the number of known captive cervid facilities in the township, or to the estimated deer density, or some combination of both factors. The number of sampled sections is constant for all selected townships and is selected completely at random or systematically.

2. Townships are selected completely at random, but sections within townships are selected with probability proportional to the risk factors in the previous example.

Another technique for incorporating suspected risk factors into the selection of SUs is to use them to allocate relative sampling effort within the strata. Consider the following examples:

1. The risk factors in the previous examples could be measured at the strata scale instead of the township scale, and strata sample size allocation, that is, the number of townships selected completely at random, is proportional to the strata risk, with an equal number of sections sampled in each selected township.

2. Strata sample size is proportional to the proximity of the strata to the nearest known CWD-positive case in an adjacent State. The number of sampled townships within each stratum is the same, but the number of sampled sections within a township is larger in strata in closer proximity to known CWD-positive deer.

Thus, there are a myriad of possibilities of sampling designs that can be used for selecting SUs. The examples detailed above provide a framework for developing a suitable sampling scheme; however, a unique and specific design needs to be developed for each study area. If possible, any sampling design created to select SUs should be based on a probabilistic sampling scheme to account for all relevant information and knowledge with regards to spatial risk factors in the study area, and the result will be good spatial distribution of the samples over the entire study area.

\section{Simulation Studies-Spatial Distribution of Samples}

In particular, the need for good spatial distribution of samples across the study area may be important, given the likely clumped or non-random distribution of CWD-infected individuals, if a surveillance program is to approach the theoretical probability of detection (Conner and Miller, 2004; Miller and Conner, 2005; Farnsworth and others, 2006; Joly and others, 2006; Osnas and others, 2009; Heisey and others, 2010). Several simulation studies have illustrated the importance of good spatial distribution in the face of non-random disease distribution and various sampling procedures.

The first example is excerpted from Samuel and others (2003) . Computer simulation was used to illustrate how spatial aggregation in disease prevalence and sampling effort can reduce the probability of detecting CWD from the idealized 
situation where CWD is randomly distributed and is randomly sampled. Specifically, they examined how the probability of detecting at least one CWD-positive individual from hunterharvested animals is affected by sample size and spatial autocorrelation in sampling effort. Based on current understanding of CWD distribution in Wisconsin, they assumed CWD prevalence was greatest in a disease cluster at the center of the landscape, and declined with distance from the center of the cluster (fig. 1).

Samuel and others (2003) used different sample sizes (50-500) and different spatial distributions of samples. They simulated the non-random nature of samples obtained from hunter-killed deer by using three levels of spatial autocorrelation: 1) "dispersed" sampling with a small degree of autocorrelation such that most of the landscape was sampled, 2) "clustered" sampling with a moderate degree of spatial autocorrelation in sampling effort, and 3) "highly clustered" sampling with a high degree of spatial autocorrelation (fig. 2A).

The probability of detecting at least one CWD-positive deer was strongly correlated with both degree of aggregation in sampling locations and number of samples (fig. $2 B$ ). The estimated detection probability from simulations converged to that predicted by random sampling when sample sizes were large (, more than 300 deer) and when sampling was evenly distributed across the landscape. However, for spatially aggregated samples the probability of detecting at least one CWD-positive deer was considerably below that expected from a truly random sample. These results emphasize the importance of uniformly sampling from SUs to achieve the expected detection probability. Local variation in deer densities, age, and sex-specific variation in CWD prevalence,

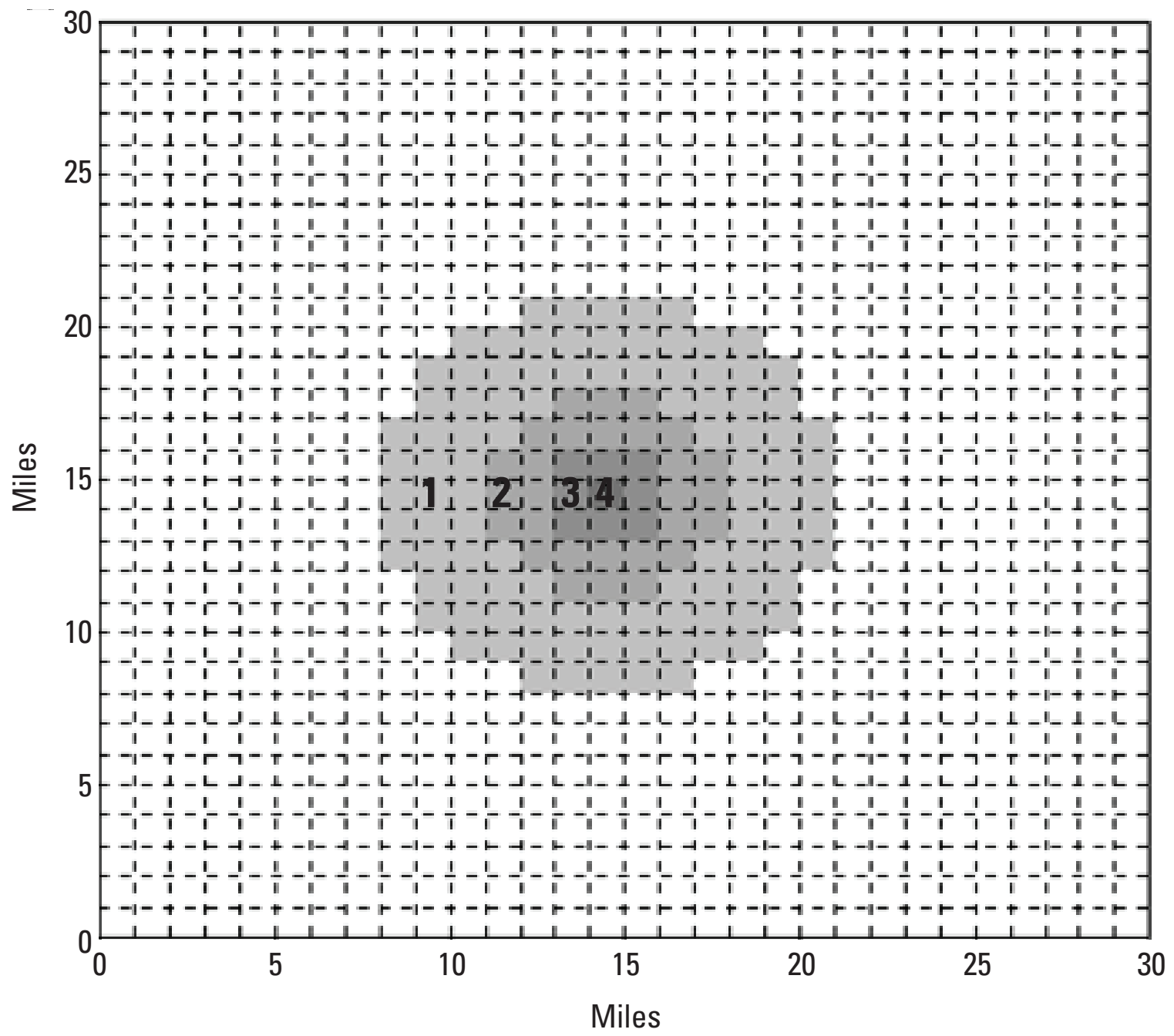

Figure 1. Landscape used in sampling simulations. Each 1-square mile cell was assumed to hold 20 deer, for a total of 18,000 deer.

\section{EXPLANATION}

Number of chronic wasting disease-positive deer in each cell 
A.
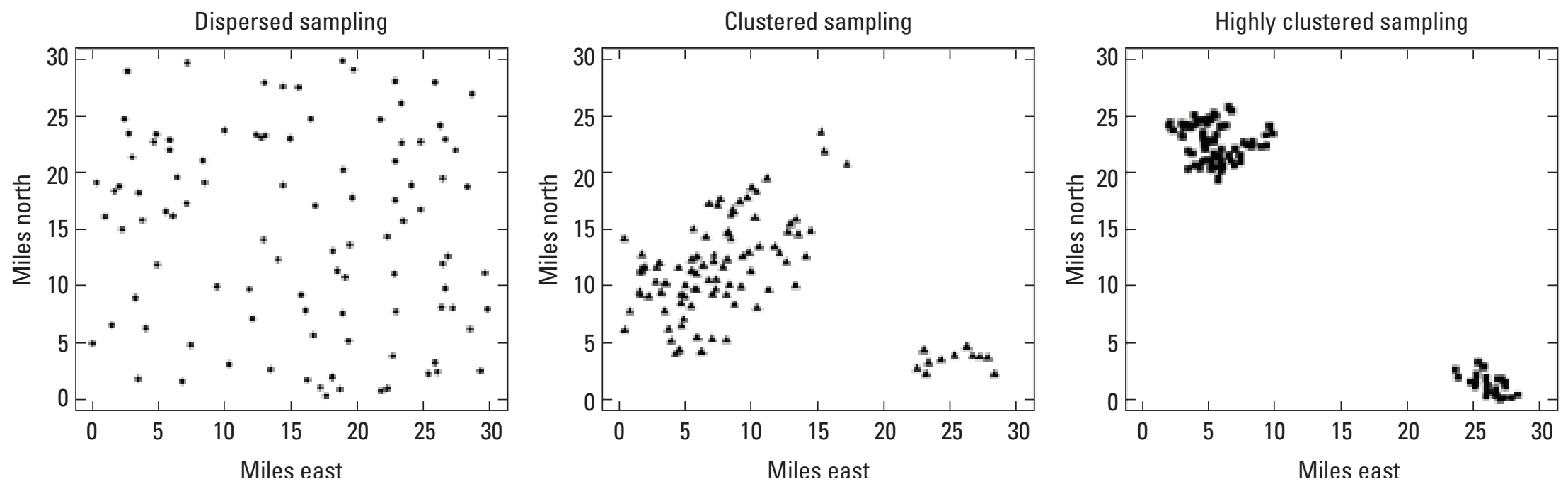

B.

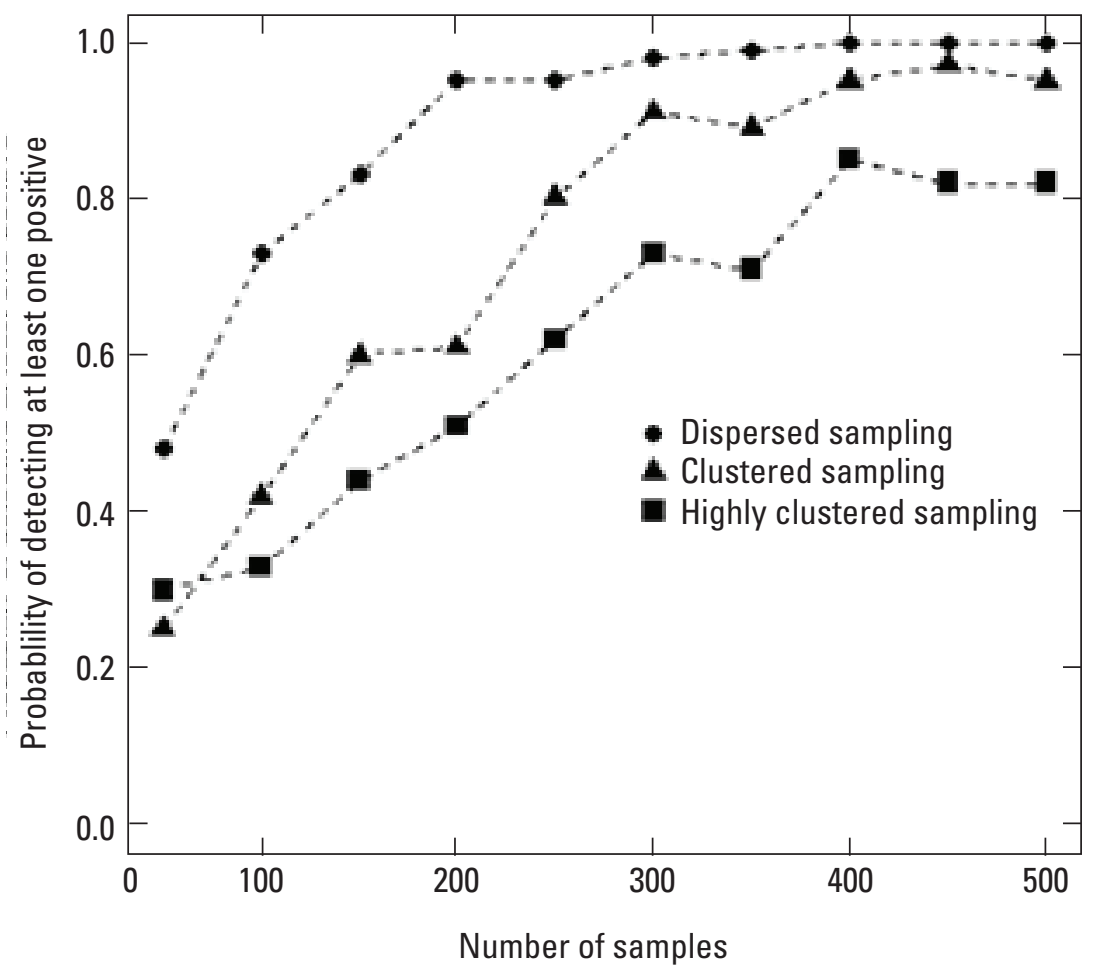

Figure 2. A, Examples of distributions of sampling locations used in the simulations. For each set of simulations, samples were taken in a distribution that was dispersed (left panel), clustered (center panel), or highly clustered (right panel). These panels correspond with the probability curves shown in figure $2 B$, matched by common symbol. $B$, The probability of detecting chronic wasting disease on a landscape as a function of sample size and dispersion of samples. 
hunter behavior, and other factors also may affect the probability of detecting CWD. However, the general pattern illustrated will likely remain true: sampling effort must be well distributed throughout the population to minimize the possibility of missing disease clusters. In addition, appropriate stratification of sampling effort with respect to suspected risk factors will increase the probability of detection of CWD.

A second simulation example is the study by Nusser and others (2008), who conducted a landscape-scale simulation study to investigate the properties of several non-probabilityand probability-based sampling designs. They used a GIS coverage of land cover in Fayette County in northeastern Iowa to distribute a population of deer based on presumed habitat associations and informed estimates of expected deer density and specified a 1.5-percent disease prevalence in the population. Diseased animals were spatially distributed either 1) completely at random, or 2) highly clustered into a single area with a few additional single spatially random outliers. Two probability-based sampling designs were evaluated: simple random sampling and stratified two-stage cluster sampling, as were two non-random/convenience designs (sampling of deer killed in collisions with vehicles (DVC) and hunter harvest). In the DVC design, diseased deer within the boundaries of a narrow roadside buffer were twice as likely to be killed by vehicles as those outside of this buffer. In the hunter-harvest design, harvest pressure, deer density, and disease prevalence were assumed to be spatially correlated, and a hunter avoidance bias was incorporated so that diseased deer had a 20-percent relative decrease in the probability of being harvested. Samples size for all designs was $n=120$.

Their results demonstrated that:

1. All sampling designs achieved the theoretical probability of detection $=0.83$ (for $n=120$ ) when diseased deer were randomly distributed. The average number of years until detection (waiting time) of at least one infected deer was 1.2 .

2. The random sampling design and the stratified cluster design maintained the theoretical probability of detection under the highly clustered disease distribution.

3. The DVC design had only a 9-percent probability of detection and an average waiting time of 11.2 years when the road system did not intersect the disease cluster.

4. The hunter selection bias in the hunter-harvest design resulted in a 7-percent absolute decrease in probability of detection and a minor increase in waiting.

Thus, these findings suggest the importance of selecting SUs from across the study area by using a probabilistic design, the danger of having a decreased probability of detecting CWD on the landscape, or an increased waiting time when all samples entering the surveillance stream are coming from convenient and spatially aggregated sources. Joly and others (2009) also provide another example of using a probabilistic design for selecting SUs in a CWD surveillance program.

\section{Selection of Population Elements from within Sampling Units-No Risk Data}

Once SUs have been selected with the desired spatial coverage and accounting for appropriate spatial risk factors, population elements (individual animals) from within these SUs must be collected and entered into the surveillance stream. If no information about demographic risk factors is available, ideally, these population elements would be collected according to some known probability sampling scheme (for example, random selection by helicopter gunning); however, in practice most samples are collected by convenience sampling (hunter harvesting). Therefore, when making inference about disease status, we are invariably forced to assume that the population from which samples are drawn is representative of the target population. The size and direction of the bias associated with this assumption is generally unknown and related to the level of dependency between the response variable (disease state) and the selection process. When disease detection is attempted, the result of having a biased selection process is only problematic if diseased animals are less likely to be included in the sample, which creates a lower detection probability than the theoretical detection probability (the probability of detecting a diseased animal at the specified prevalence is less than $1-\alpha$, where $1-\alpha$ is the theoretical detection probability). For example, if hunters harvest infected animals at the same rate as healthy individuals, bias in the sample from this group is negligible because the selection of individuals is not dependent on disease status. However, because the dependency is unknown and, in general, bias will affect our probability of detecting a diseased individual, we should attempt to minimize it by applying probabilistic sampling schemes when possible.

In most CWD surveillance systems, samples are collected from multiple sources, some of which involve active collection by agency personnel by culling, collection of deer killed in collisions with vehicles, and so forth, and others that enter through passive collection (for example, hunter submissions). For samples that are actively collected, we strongly urge collecting population elements by using a probabilistic scheme whenever possible to ensure the realized disease detection probability is as close as possible to the theoretical detection probability. In addition, partial implementation of probability designs such as espoused here, where SUs are selected in a probabilistic manner and population elements are selected by convenience sampling, will help to reduce the bias associated with using a non-probability sample of population units, but will not eliminate it. Practitioners must be aware of this shortcoming. Nusser and others (2008) provide some insight into the effect of selection bias on disease detection probability and waiting time. 


\section{Selection of Population Elements from within Sampling Units-Risk Data Available}

For diseases such as CWD that have been studied, information often is available regarding demographic risk factors (see previous chapter). This information can be exploited to increase disease detection probability. By employing unequal probability sampling, such as PPS, in selection of individual animals within the SUs, we can increase the sampling rate within segments of the population with the greatest risk or highest prevalence, and thereby increase our probability of detecting CWD. The underlying motivation for this approach is to optimally allocate limited resources by sampling population elements based on disease risk. This assumes a fixed budget for sampling and that high risk animals are no more costly to sample. For example, prevalence in suspect animals that exhibit clinical signs of CWD is considerably higher than prevalence in hunter-harvested individuals (Miller and others, 2000). By "over-sampling" suspect animals, therefore, our probability of detecting CWD, if present, will be greater, and waiting time until first detection will be reduced compared to sampling exclusively from hunter-harvested animals, assuming the same total sample sizes in either case. This principle is the basis for the weighted surveillance system (Walsh and Miller, 2010).

Ideally, all the samples entering the surveillance stream should come from the segment of the population with the highest risk; however, in practice this is highly problematic, because there will be a limited number of CWD suspect animals in the population. Thus, acquiring a sufficient number of samples from this population segment may not be practical or feasible, and samples from other segments must be collected. Collection from the remaining segments should be done with respect to their risk (focusing on the highest risk groups first) to attain the underlying surveillance goal: achieving the theoretical disease detection probability with the fewest number of samples or least cost.

Within a subpopulation, individuals should be sampled according to some probabilistic scheme. But, in reality the samples being collected come from various population segments and will be attained by convenience sampling. Although, as described above, convenience sampling creates biased detection probabilities, nevertheless Walsh and Miller (2010) demonstrated by simulation that disease detection probabilities were acceptable by using the weighted surveillance scheme, even in the face of moderately biased weights as would arise from individual heterogeneity (each individual has its own unique probability of entering the surveillance stream), or convenience sampling (Diefenbach and others, 2004). Thus, when adequate information regarding demographic risk factors is available, surveillance activities can exploit this data to increase overall efficiency and effectiveness.

As an aside, we strongly recommend that whether an agency employs a weighted surveillance scheme or not, all known clinical suspect animals should be collected by agency personnel and submitted to proper diagnostic labs for necropsy. These individuals can provide critical information regarding and, possibly, early detection of a wide-range of wildlife diseases in addition to CWD. Therefore, suspect animals are a valuable tool for monitoring overall population health and viability and should be exploited as such.

\section{Sample Size}

Once an agency decides to implement a weighted surveillance scheme, the question then becomes how many samples are required. Sample size decisions are driven by the survey design and the specific statistical estimation objective. Most commonly, a statistical objective is stated in terms of desired precision of the estimator of a population characteristic, such as the mean or total for the target population. In a detection surveillance program, the statistical objective is to make an inference that "if the prevalence of CWD in animals in the target population is at least $X$ percent, then we are $Y$-percent confident ${ }^{1}$ that our surveillance plan will detect at least one infected animal."

The difficulty when employing the weighted surveillance scheme is determining the number of population elements within and across SUs that need to be collected to designate an area as disease-free. Generally, sampling from an area continues until an adequate number of samples is collected to ensure the theoretical probability of detecting the disease is at least equal to some statistical power when the disease in the target population occurs at some user-specified prevalence. These samples come from various subpopulations with varying disease risks. Therefore, to employ the weighted surveillance system and determine needed sample sizes, weights, which incorporate risk or prevalence information, have to be calculated for each subpopulation of interest. Historically, each individual entering the surveillance stream received the same weight (1); however, this is inefficient if only a limited number of samples can be examined.

The first step in employing a weighted surveillance system is to estimate the weights associated with various demographic groups of interest. In simplest terms, these weights are a measure of worth of each demographic group for detecting CWD compared to some baseline demographic group (the group of animals to which the design prevalence applies and whose weight is defined to be 1). These weights are commonly used metrics of relative risk or hazard ratios in survival analyses. Walsh and Miller (2010) describe a maximum likelihood methodology for calculating these weights based on Poisson approximation to the binomial and designating harvested adult males as the baseline population. We employed a recently developed methodology by using a Bayesian approach to estimating "real weights" that allows for incorporation of variability in the hazard ratios estimated for each population segment (Chris Jennelle, written commun,

\footnotetext{
${ }^{1}$ Note, in the statistical context this would be described as power of the study design or test, whereas confidence is associated with a measure of precision of an estimate generated from data that has already been gathered.
} 
2012). This accounting for variability is lacking in the Walsh and Miller (2010) approach due to difficulties in addressing the issue within the frequentist framework; however, the methods produce similar results when the appropriate population segment is chosen as the baseline and the weights are precisely estimated (Chris Jennelle, written commun, 2012).

We used this Bayesian technique to estimate "real weights" (hereafter referred to just solely as weights) for two species: elk and mule deer. Data collected from data analysis units (DAUs) known to contain CWD in Colorado during 2003-6 were used to estimate the elk and mule deer weights. We used 20,400 mule deer samples and 21,946 elk samples for this analysis. We stratified the submitted cases for both elk and mule deer into eight groups:

1. and 2. clinical CWD "suspects," female and male more than 1-year old; 3.-7. harvested animals: adult males, adult females, yearling males, yearling females, or fawns (young of the year of either sex); and 8. all other mortalities, such as road-kills, predation mortalities, and other deaths for both sexes and ages, except fawns.

For elk, harvested yearling males and calves were assigned a weight of zero, because no positive cases were found in these groups during the years of 2003-6. When using a weighted surveillance system, a baseline population segment to which the specified design prevalence applies must be determined. This population segment is the demographic group from which all other groups are scaled; that is, they will have a weight of 1 in our surveillance system. Although any segment can arbitrarily be selected as the baseline, we recommend that it be the population stratum for which the sample sizes are consistently the largest, and it should also be chosen with herd management goals in mind. Our baseline population segment (the segment of the population to which the specified design prevalence applies) was harvested adult males for mule deer and harvested adult females for elk. We chose these groups because they are consistently the most represented in our surveillance stream and are a focus of our herd management strategies for these species.

By using the Bayesian software WinBUGS (Lunn and others, 2000), we calculated the mean, median and the 95-percent credible intervals of the hazard ratio for each population segment of each species (tables 1 and 2). From these hazard ratios, we calculated the weight for each demographic group (tables 3 and 4). As expected, clinical suspect mule deer had the highest weight, followed by the "other" population segment, adult-harvested animals, yearling-harvested animals, and young-of-the-year. Elk showed the same general rankings; however, the "other" group had a lower weight than the harvested adult population segments. Similar patterns and weightings have been shown in white-tailed deer as well (Chris Jennelle, written communication, 2012). This ranking is intuitive, and a higher weight is associated with individuals from population strata with higher estimated prevalence. The largest difference between the estimated hazard ratios and weights were associated with groups of animals with small sample sizes. Baseline prevalence estimates varied from 0.027 to 0.037 in mule deer and from 0.0074 to 0.0092 in elk. We also calculated the number of samples needed from each population segment if all the samples entering the surveillance stream arose from that subpopulation (tables 3 and 4).

Table 1. Estimated mean hazard ratios, associated standard deviations, Markov Chain Monte Carlo (MCMC) errors, and quantiles for various demographic groups of mule deer (Odocoileus hemionus) based on samples collected from chronic wasting disease-positive areas in Colorado during 2003-6.

\begin{tabular}{lcccccc}
\hline \multicolumn{1}{c}{ Group } & Mean & $\begin{array}{c}\text { Standard } \\
\text { deviation }\end{array}$ & $\begin{array}{c}\text { Markov Chain } \\
\text { Monte Carlo } \\
\text { error }\end{array}$ & $\begin{array}{c}\mathbf{2 . 5 0} \\
\text { percent }\end{array}$ & Median & $\begin{array}{c}\mathbf{9 7 . 5 0} \\
\text { percent }\end{array}$ \\
\hline Suspect female & 14.13 & 2.390 & 0.0151 & 9.90 & 13.97 & 19.24 \\
Suspect male & 12.19 & 2.070 & 0.0135 & 8.50 & 12.06 & 16.64 \\
Other & 1.93 & 0.245 & 0.0018 & 1.49 & 1.92 & 2.44 \\
Harvest_adult males & 1.00 & $\mathrm{NA}$ & $\mathrm{NA}$ & $\mathrm{NA}$ & $\mathrm{NA}$ & $\mathrm{NA}$ \\
Harvest-adult females & 0.57 & 0.065 & 0.0005 & 0.46 & 0.57 & 0.71 \\
Harvest-yearling females & 0.44 & 0.150 & 0.0009 & 0.20 & 0.43 & 0.78 \\
Harvest-yearling males & 0.25 & 0.077 & 0.0004 & 0.12 & 0.24 & 0.43 \\
Harvest-fawns & 0.03 & 0.032 & 0.0002 & 0.001 & 0.02 & 0.12 \\
\hline
\end{tabular}


Table 2. Estimated mean hazard ratios, associated standard deviations, Markov Chain Monte Carlo (MCMC) errors, and quantiles for various demographic groups of elk (Cervus elaphus) based on samples collected from chronic wasting disease-positive areas in Colorado during 2003-6.

\begin{tabular}{lcccccc}
\hline \multicolumn{1}{c}{ Group } & Mean & $\begin{array}{c}\text { Standard } \\
\text { deviation }\end{array}$ & $\begin{array}{c}\text { Markov Chain } \\
\text { Monte Carlo } \\
\text { error }\end{array}$ & $\begin{array}{c}\mathbf{2 . 5 0} \\
\text { percent }\end{array}$ & Median & $\begin{array}{c}\mathbf{9 7 . 5 0} \\
\text { percent }\end{array}$ \\
\hline Suspect female & 29.25 & 11.920 & 0.045 & 11.23 & 27.44 & 57.26 \\
Suspect male & 21.28 & 12.830 & 0.048 & 4.24 & 18.72 & 53.08 \\
Other & 1.06 & 0.633 & 0.002 & 0.21 & 0.94 & 2.62 \\
Harvest-adult males & 1.28 & 0.264 & 0.001 & 0.83 & 1.25 & 1.86 \\
Harvest-adult females & 1.00 & $\mathrm{NA}$ & $\mathrm{NA}$ & $\mathrm{NA}$ & $\mathrm{NA}$ & $\mathrm{NA}$ \\
Harvest-yearling females & 0.46 & 0.244 & 0.001 & 0.12 & 0.42 & 1.06 \\
\hline
\end{tabular}

Table 3. The "real weights" that account for uncertainty in the estimates of the hazard ratios and associated number of samples (N) needed to reach a 95-percent confidence that an area of interest is "disease-free" if the prevalence is less than or equal to 0.01 for mule deer (Odocoileus hemionus) from chronic wasting disease-positive areas in Colorado during 2003-6.

\begin{tabular}{lcr}
\hline \multicolumn{1}{c}{ Group } & Weight & $\begin{array}{c}\text { Number of } \\
\text { samples } \\
\text { (N) }\end{array}$ \\
\hline Suspect female & 13.60 & 22 \\
Suspect male & 11.50 & 26 \\
Other & 1.90 & 158 \\
Harvest—adult males & 1.00 & 300 \\
Harvest—adult females & 0.56 & 536 \\
Harvest-yearling females & 0.33 & 909 \\
Harvest-yearling males & 0.19 & 1579 \\
Harvest-fawns & 0.001 & 300,000 \\
\hline
\end{tabular}

Table 4. The "real weights" that account for uncertainty in the estimates of the hazard ratios and associated number of samples (N) needed to reach a 95-percent confidence that an area of interest is "disease-free" if the prevalence is less than or equal to 0.01 for elk (Cervus elaphus) from chronic wasting disease-positive areas in Colorado during 2003-6.

\begin{tabular}{lcc}
\hline \multicolumn{1}{c}{ Group } & Weight & $\begin{array}{c}\text { Number of } \\
\text { samples } \\
\text { (N) }\end{array}$ \\
\hline Suspect female & 18.75 & 16 \\
Suspect male & 8.57 & 35 \\
Other & 0.41 & 725 \\
Harvest_adult males & 1.16 & 259 \\
Harvest_adult females & 1.00 & 300 \\
Harvest_yearling females & 0.23 & 1,310 \\
\hline
\end{tabular}


Once the weights have been calculated, we need to determine the total number of samples needed from each of the population segments. The problem is that rarely do we have control over when and from what risk groups the samples entering the CWD surveillance stream are collected, which makes having preset sample sizes for each group infeasible. One solution is a "points system." With this system, regardless of how the population elements are selected, once an element enters the surveillance stream it is awarded a specified number of "points." Point values assigned to each individual sample equal the estimated weights described above. Samples continue to enter the surveillance stream until total accumulation of points equals some target value. Thus, this points-based system provides an intuitive approach to disease surveillance that can be readily implemented by field personnel. This type of system was first espoused by Cannon (2002) and later developed for BSE surveillance (Wilesmith and others, 2004), with most recent application to CWD (Walsh and Miller, 2010).

Under this points system, the target value $(n)$, which is the number of points that need to be accumulated during surveillance activities, must be specified for each SU. It is based on the desired confidence $(\alpha)$ and the predetermined design prevalence (the minimum prevalence level in the baseline group at which the disease is to be detected; $p_{\text {design }}$ ). It is calculated as follows:

$$
n=\frac{-\ln (1-\alpha)}{p_{\text {design }}}
$$

This equation is recognizable as the standard equation for calculating the number samples needed to demonstrate freedom from disease, assuming the number of positive cases follow a Poisson distribution (Dohoo and others, 2003). An alternative when population size is known and relatively small is to use the hypergeometric distribution (Samuel and others, 2003); however, both formulations provide nearly identical results for population sizes of ungulates likely to be encountered in practice, and thus we will only discuss the simpler Poisson model. A Bayesian approach can also be taken to estimate the target value; however, the values are once again nearly identical to those derived from the Poisson model.

We will now provide a simple example to illustrate the use and benefit of the weighted surveillance system within a SU. For purposes of this example, the total population of the SU is estimated as 10,000 mule deer. It was predetermined that we wished to detect the presence of CWD when the number positive deer in the population was greater than or equal to $(\geq) 100$. Thus, the design prevalence was set at 0.01 and $\alpha=0.95$, which resulted in a target value for the SU of 300. This means we desire a 95-percent probability of detecting disease if prevalence is 1 percent or greater. Samples were collected until the target value was reached by using the weights for mule deer from table 3 to assign points. The following samples were collected: 17 harvested yearling females,
12 harvested yearling males, 5 CWD-suspect males, 4 CWDsuspect females, 105 harvested adult males (the baseline population segment), 21 road-killed or predator-killed deer, and lastly 63 harvested adult females. A total of 227 deer were submitted into the surveillance stream to reach the target value. Thus, use of the weighted surveillance system reduced the required sample size by 73 animals, which represents a cost savings for this single SU of approximately $\$ 5,525$, assuming the cost of testing is approximately $\$ 75$ per animal. When multiple SUs are sampled, the cost savings increase significantly. In addition to showing the benefit of using a weighted surveillance system, this example also illustrates the importance of understanding the implication of setting the target value. For example, if we designate an entire state as an SU and the design prevalence is set at 0.01 with $\alpha=0.95$, then by using the above equation $n=300$. Thus, once the total points from samples entering the surveillance stream equal 300, we will be 95-percent confident the disease prevalence is less than or equal to $(\leq) 0.01$. However under this scenario, if the total population size is $1,000,000$, this implies that there may be as many as 10,000 CWD-positive individuals in the SU, which is likely well above the threshold for what is acceptable to most agencies. Therefore, each agency should first determine the number of positive individuals they are willing to allow to go undetected in each SU, and then set the design prevalence and the resulting target values accordingly.

Once the target value for each SU is specified and the weights are calculated, the final step is to determine the number of SUs to sample. It is clear that standard approaches to sample size determination, such as use of a binomial probability argument based on the assumption of completely random sampling of individual deer for measurement (Samuel and others 2003,) are inadequate for supporting informed decisions about SU selection within complex CWD surveillance programs. This difficulty is fairly common in complex estimation procedures used in wildlife population biology, and in such cases biometricians turn to computer simulation experiments to investigate and compare statistical performance under a variety scenarios specified by assumptions about the biological and sampling processes of interest. For CWD surveillance designs, evaluation of non-probabilistic or partially probability-based approaches for SU selection can also be evaluated. We encourage the use of simulation modeling to inform State agencies about the relative costs and effects associated with various sample sizes and distributions of SUs. Examples of such simulation modeling were previously detailed. Although we cannot provide specific guidance on the appropriate sample size of the SUs because each jurisdiction will vary in its requirements; nevertheless, as previously described, enough SUs must be selected to ensure adequate spatial distribution. For example, in Colorado animals are submitted for surveillance from all data analysis units (DAUs are SUs for Colorado) across the State with a target value of 300 points for each DAU. 


\section{Temporal Scale of Disease Surveillance}

As resources have become more limiting, agencies have accordingly become more interested in conducting periodic as opposed to continuous (annual) disease surveillance. One advantage of periodic surveillance is the ability for funding sources to allocate additional resources in a cyclic manner to agencies during years of surveillance while allocating resources to other agencies in years when surveillance is not being conducted. The benefit of this type of allocation is more intensive surveillance efforts (increased sampling activities) can be conducted during surveillance years with the additional resources. This should allow for more sampling and/or population units to enter the surveillance stream, increasing confidence that a jurisdiction is below the specified design prevalence. However, social and political considerations may require an annual surveillance effort. In addition, operational considerations may make periodic surveillance problematic. For example, it may be difficult or more costly to mobilize and maintain the needed personnel and infrastructure for periodic surveillance than if surveillance is conducted annually. Also, if an agency is relying on passive sampling, such as hunter submissions, as a major component of surveillance, then sample submission may decrease when collection doesn't occur on an annual basis. Lastly, with periodic surveillance, there is an increased probability that if CWD becomes established it may go undetected for a longer period, which has implications on control efforts. One possible periodic sampling scheme, which may alleviate some of the aforementioned operational, social, and political considerations yet still reduce costs, is to conduct surveillance annually, while rotating the geographic region at which is surveillance is conducted. Regardless of the design of the periodic surveillance system, if an agency intends to employ disease surveillance over varying time scales, it must carefully weigh the risks associated with this approach compared to an annual surveillance system. If a periodic surveillance system is instituted, the lag period between surveillance activities should be based on biological and epidemiological characteristics of the study species and CWD.

Another temporal consideration is whether or not multiple-year inference can be used to make surveillance more efficient. One form of multiyear inference in surveillance programs is expected waiting time to first detection, or more formally, "for a specified surveillance design conducted annually, what is the expected number of years until the first detection, assuming a disease prevalence of $X$ percent?" This question is of particular relevance under the philosophy that a detection surveillance program could be viewed in a cumulative, multiyear framework, in which data from annual surveys is formally accumulated into a statistical framework that allows multiyear inference about waiting time. A consequence of this approach might be that annual cost would be reduced when compared to a design framework in which inferences are made independently in each year, because reduction in sample size or incomplete sampling of the target population in a given year might still result in acceptable statistical confidence for multiyear inference. Readers interested in this approach to multiyear inference are referred to Nusser and others (2008).

A second type of multiyear inference is described by Hadorn and others (2002). With this technique, the critical assumption is that the probability of a CWD-infected individual entering the system either from immigration of freeranging animals or by movement of captive animals is known or can be estimated. It is important when employing multiyear inference approaches, or any surveillance system, that the assumptions, advantages, and disadvantages of the various techniques be carefully evaluated and their implications for management and disease control be understood. Readers are referred to the aforementioned article for more information.

\section{When Is Enough...Enough?}

Another common question is "when have I conducted enough surveillance to confidently believe my jurisdiction is disease-free?" Bohning and Greiner (2006) provide a statistical framework for estimating the smallest number of samples required over multiple survey events, which need to be tested to ensure an area is free of disease for a given design prevalence and power. Their approach is based on a geometric distribution for waiting time (that is, the time until first detection of disease) and was developed for surveillance of BSE. An extension of this framework allows for heterogeneity in design prevalence, which would be particularly useful for CWD surveillance.

Several factors need to be considered when proposing to discontinue disease surveillance in addition to assumptions of the above model. First, what is the risk of an infected individual entering the study area through immigration of free-ranging animals from known or unknown positive jurisdictions or movement of captive cervids? If this risk is greater than zero, decisions on whether or not to continue to conduct surveillance should carefully weigh the level of this risk, because ultimately some chance exists of introduction of the disease. Secondly, agencies need to consider if they have adequate spatial coverage of sampling units across the jurisdiction to ensure all potential pockets of disease are sampled, and if they have effectively sampled individual animals within sampling units. Third, agencies need to consider the social and political repercussions of stopping surveillance activities. Lastly, availability of resources will play perhaps the most important role in determining whether surveillance should proceed or not. A cautionary tale against stopping surveillance too soon is provided by past CWD developments in Michigan. CWD surveillance in Michigan had continued since 1998 with 23,739 white-tailed deer, $887 \mathrm{elk}$, and 46 moose tested as of October 21, 2008 (Daniel O’Brien, Michigan Department of Natural Resources, oral commun). In addition, 8,452 captive cervids have been tested by the Michigan Department of Agriculture. 
Even with 10 years of surveillance effort, the first positive case was discovered in a captive animal in fall of 2008. Thus, even with a reasonable level of surveillance effort for a considerable period of time, a new case may be detected where a jurisdiction was previously believed to be CWD-free when the probability of introduction is greater than zero.

\section{Conclusion}

In this chapter, we illustrated how a weighted surveillance system can be used to effectively and efficiently conduct CWD surveillance. This surveillance system is based on a number of assumptions, which need to be met if the system is to achieve the theoretical detection probability (Walsh and Miller 2010; Chris Jennelle, written commun, 2012). Understanding these assumptions and the impact of the failure to meet these assumptions is critical when implementing this and any surveillance strategy. We also recognize the potential for species differences, spatial and temporal variability in CWD disease dynamics may affect the estimated weights and strongly recommend each agency develop its own weights for their region and species of interest whenever possible. However, for regions where CWD has not yet been detected and data are not available to estimate the weights, we recommend using the weights provided in tables 3 and 4 for the appropriate species. In lieu of available data, we believe that this is a reasonable approach because the various risk factors (age, sex, and so forth) upon which the weights are derived have shown consistent patterns in the epidemiology of CWD throughout its range and across time (Joly and others, 2003; Conner and Miller, 2004; Miller and Conner, 2005; Farnsworth and others, 2006; Grear and others, 2006; Joly and others, 2006; Osnas and others, 2009; Grear and others, 2010; Heisey and others, 2010; Walsh and Miller, 2010), which would indicate that the observed differences in risk used to calculate the weights, arise largely from underlying disease processes rather than local processes that may vary considerably across locales. For example, accounting for the different baseline population segments used in analysis, the estimated weights for mule deer in Colorado are generally similar to those estimated for whitetailed deer in Wisconsin. These represent two different species from two regions with vastly different histories of CWD and varying environmental characteristics (Walsh and Miller 2010; Chris Jennelle, written commun, 2012). This provides additional credence to the assertion that temporal and spatial differences in the weights may exist, but those differences are not expected to be substantial. In addition, the Bayesian technique used to estimate the weights presented herein explicitly accounts for the variability within data from which the weights are derived, which addresses concerns of withinsite temporal variability. Also, it is important to note that even if differences between the weights used in surveillance and the true underlying weights do exist, as long as these differences do not cause significant bias in the resulting weights (that is, $\geq 30$ percent), the overall effect on probability of detecting CWD-positive individuals is likely minimal (Walsh and Miller, 2010). Therefore, because the ultimate goal of surveillance is early disease detection, we believe that using the weighted surveillance is preferred over the traditional surveillance approach even if the estimated weights employed in the surveillance efforts are somewhat different than the true underlying weights for a jurisdiction.

We believe the two-stage surveillance scheme presented herein, which is based on sampling SUs and subsequently population elements within SUs, provides a powerful and costeffective tool for conducting disease surveillance. By accounting for both spatial and demographic risk factors in the design of the surveillance system, we are able to effectively utilize this information to create a more efficient surveillance system. In addition, using a points approach to create a weighted surveillance system provides an intuitive surveillance mechanism, which can be easily employed by field personnel. With limited resources available to agencies to conduct disease surveillance, we believe the surveillance system described herein represents the next step in the evolution of surveillance for CWD.

\section{Acknowledgments}

We would like to acknowledge Dr. Dennis M. Heisey for his thoughts, contributions and helpful suggestions during the development of this chapter.

\section{Literature Cited}

Bohning, D., and Greiner, M., 2006, Modeling cumulative evidence for freedom from disease with applications to BSE surveillance trials: Journal of Agricultural, Biological, and Environmental Statistics, v. 11, p. 280-295.

Cannon, R.M, 2002, Demonstrating disease freedom-Combining confidence levels: Preventive Veterinary Medicine, v. 52 , p. $227-249$.

Conner, M.M., and Miller, M.W., 2004, Movement patterns and spatial epidemiology of a prion disease in mule deer population units: Ecological Applications, v. 14, p. 18701881.

Diefenbach, D.R.; Rosenberry, C.S.; and Boyd, R.C., 2004, From the field: Efficacy of detecting chronic wasting disease via sampling hunter-killed white-tailed deer: Wildlife Society Bulletin, v. 32, p. 267-272. 
Doherr, M.G.; Heim, D.; Fatzer, R.; Cohen, C.H.; Vandevelde, M.; and Zurbriggen, A., 2001, Targeted screening of high-risk cattle populations for BSE to augment mandatory reporting of clinical suspects: Preventive Veterinary Medicine, v. 51, p. 3-16.

Dohoo, I.; Martin, W.; and Stryhnn, H., 2003, Veterinary Epidemiologic Research: Charlottetown, Canada, AVC, Inc., $706 \mathrm{p}$.

Farnsworth, M.L.; Hoeting, J.A.; Hobbs, N.T.; and Miller, M.W., 2006, Linking chronic wasting disease to mule deer movement scales: A hierarchical Bayesian approach: Ecological Applications, v. 16, p. 1026-1036.

Grear, D.A.; Samuel, M.D.; Langenberg, J.A.; and Keane, D., 2006, Demographic patterns and harvest vulnerability of chronic wasting disease infected white-tailed deer in Wisconsin: Journal of Wildlife Management, v. 70, p. 546-533.

Grear, D.A.; Samuel, M.D.; Scribner, K.T.; Weckworth, B.V.; and Langenberg, J.A., 2010, Influence of genetic relatedness and spatial proximity on chronic wasting disease infection among female white-tailed deer: Journal of Applied Ecology, v. 47 , p. $532-540$.

Hadorn, D.C.; Rufenacht, J.; Hauser, R.; and Stark, K.D., 2002, Risk-based design of repeated surveys for the documentation of freedom from non-highly contagious diseases: Preventive Veterinary Medicine, v. 56, p. 179-192.

Heisey, D.M.; Osnas, E.E.; Cross, P.C.; Joly, D.O.; Langenberg, J.A.; and Miller, M.W., 2010, Linking process to pattern: Estimating spatiotemporal dynamics of a wildlife epidemic from cross-sectional data: Ecological Monographs, v. 80, p. 221-240.

Joly, D.O.; Ribic, C.A.; Langenberg, J.A.; Beheler, K.; Batha, C.A.; Dhuey, B.J.; Rolley, R.E.; Bartelt, G.; Van Deelen, T.R.; and Samuel, M.D., 2003, Chronic wasting disease in free-ranging Wisconsin white-tailed deer: Emerging Infectious Diseases, v. 9, p. 599-601.

Joly, D.O.; Samuel, M.D.; Langenberg, J.A.; Blanchong, J.A.; Batha, C.A.; Rolley, R.E.; Keane, D.P.; and Ribic, C.A., 2006, Spatial epidemiology of chronic wasting disease in Wisconsin white-tailed deer: Journal of Wildlife Diseases, v. 42 , p. $578-588$.

Joly, D.O.; Samuel, M.D.; Langenberg, J.A.; Rolley, R.E.; and Keane, D.P., 2009, Surveillance to detect chronic wasting disease in white-tailed deer in Wisconsin: Journal of Wildlife Diseases, v. 45, p. 989-997.

Lohr, S.L., 1999, Sampling design and analysis: Pacific Grove, Calif., Duxbury Press.
Lunn, D.J.; Thomas, A.; Best, N.; and Spiegelhalter, D., 2000, WinBUGS-A Bayesian modelling framework: Concepts, structure, and extensibility: Statistics and Computing, v. 10, p. 325-337.

Miller, M.W., and Conner, M.M., 2005, Epidemiology of chronic wasting disease in free-ranging mule deer: Spatial, temporal, and demographic influences on observed prevalence patterns: Journal of Wildlife Diseases, v. 41, p. 275-290.

Miller, M.W.; Williams, E.S.; McCarty, C.W.; Spraker, T.R.; Kreeger, T.J.; Larsen, C.T.; and Thorne, E.T., 2000, Epizootiology of chronic wasting disease in free-ranging cervids in Colorado and Wyoming: Journal of Wildlife Diseases, v. 36 , p. 676-690.

Nusser, S.M.; Clark, W.R.; Otis, D.L.; and Huang, L., 2008, Sampling considerations for disease surveillance in wildlife populations: Journal of Wildlife Management, v. 72, p. 52-60.

Osnas, E.E.; Heisey, D.M.; Rolley, R.E.; and Samuel, M.D., 2009, Spatial and temporal patterns of chronic wasting disease: Fine-scale mapping of a wildlife epidemic in Wisconsin: Ecological Applications, v. 19, p. 1311-1322.

Samuel, M.D.; Joly, D.O.; Wild, M.A.; Wright, S.D.; Otis, D.L.; Werge, R.W.; and Miller, M.W., 2003, Surveillance strategies for detecting chronic wasting disease in free-ranging deer and elk: Results of a CWD surveillance workshop, 10-12 December 2002: U.S. Geological Survey National Wildlife Health Center, 40 p., accessed May 27, 2011, at http://www.nwhc.usgs.gov/publications/fact_sheets/pdfs/ cwd/CWD_Surveillance_Strategies.pdf.

Stark, K.D.; Regula, G.; Hernandez, J.; Knopf, L.; Fuchs, K.; Morris, R.S.; and Davies, P., 2006, Concepts for risk-based surveillance in the field of veterinary medicine and veterinary public health: Review of current approaches: BMC Health Services Research, v. 6, p. 20.

Thurmond, M.C, 2003, Conceptual foundations for infectious disease surveillance: Journal of Veterinary Diagnostic Investigation, v 15, p. 501-514.

Walsh, D.P., and Miller, M.W., 2010, Designing a weighted surveillance system for chronic wasting disease in Colorado: Journal of Wildlife Diseases, v. 46, p. 118-135.

Wilesmith, J.; Morris, R.; Stevenson, M.; Cannon, R.; Prattley, D.; and Benard, H., 2004, Development of a method for evaluation of national surveillance data and optimization of national surveillance strategies for bovine spongiform encephalopathy: Weybridge, United Kingdom, European Union TSE Community Reference Laboratory, 34 p. 



\title{
Chapter 3 \\ Monitoring Surveillance: Disease Investigations in Chronic Wasting Disease-Positive Areas
}

\author{
By Julie A. Langenberg, VMD', Daniel J. O'Brien, DVM, Ph.D. ${ }^{2}$, Daniel P. Walsh, Ph.D. ${ }^{3}$, \\ Lynn H. Creekmore, DVM ${ }^{4}$, Jenny G. Powers, DVM, Ph.D. ${ }^{5}$, and Michael W. Miller, DVM, Ph.D. ${ }^{6}$
}

\section{Introduction}

After chronic wasting disease (CWD) detection, surveillance goals may shift to disease monitoring. The first step in monitoring is usually to assess and establish a baseline for the intensity of disease occurrence and/or its spatial distribution on the landscape. Once this initial baseline assessment is completed, further monitoring surveillance then can be tailored as needed to address one or more of the following questions:

- "Is there change in the prevalence of disease over time?"

- "Is there change in the rate of disease transmission or force of infection over time?"

- "Is there a change in the spread or contraction of CWD over the landscape over time?"

- "What are the most important means of transmission and spread in the population?"

- "What are the impacts of management actions on epidemic dynamics and/or geographic spread over time?"

Determining the specific goals of monitoring efforts and formulating specific questions to be answered are critical to collecting useful surveillance data. Moreover, determining the magnitude or type of change to be detected in the pathogen, the host(s), or the environment is important to consider when planning monitoring actions to collect the data necessary to measure such changes.
Although approaches for CWD detection surveillance are relatively well defined here and elsewhere (Samuel and others, 2003), surveillance intended primarily to monitor trends represents an arena of considerably less experience, guidance, and certainty of outcome. Consequently, investing some effort toward designing and conducting monitoring programs in a rigorous and scientifically valid fashion to collect the highest quality data possible will provide useful information to advance our understanding about the most effective monitoring approaches in addition to gathering the trend data themselves.

Monitoring to detect changes in disease patterns should be particularly valuable when linked with research to understand the epidemiology of CWD. Striving to link monitoring programs with research objectives can create a synergistic effect that may provide more informative data than would be obtained if these activities occurred in isolation. Monitoring is also an integral component of agency programs designed to manage CWD. Monitoring changes in disease patterns and impacts of disease on susceptible populations provides the primary source of information for assessing the effect of management programs and is crucial for evaluating population responses to adaptive management approaches for CWD.

In shifting program emphasis from surveillance for disease detection to monitoring for disease trends, the complexity of surveillance strategies, methods, and analyses generally increases. Some components of a surveillance program are common to all of the surveillance objectives; for example, if the main question concerns spread of CWD to new locations on the landscape, detection strategies necessarily will be part of the approach.

\footnotetext{
${ }^{1}$ Wisconsin Department of Natural Resources, currently affiliated with the International Crane Foundation.

${ }^{2}$ Michigan Department of Natural Resources.

${ }^{3}$ Colorado Division of Wildlife, currently affiliated with the U.S. Geological Survey, National Wildlife Health Center.

${ }^{4}$ U.S. Department of Agriculture-APHIS.

${ }^{5}$ National Park Service.

${ }^{6}$ Colorado Division of Wildlife.
} 
In formulating a plan for CWD monitoring surveillance, it is important to consider some broad principles and how they apply to a specific jurisdiction and the disease patterns observed:

1. Choices about how to monitor CWD trends in freeranging populations should not only be defined by the questions to answer, but also with consideration of what ultimately will be done with monitoring results. Among other uses, trend data may be of value in (1) deciding if, to what degree, and how CWD control will be attempted; (2) helping to target control actions such as culling;

(3) implementing risk management by local and neighboring jurisdictions, for example, carcass movement restrictions, feeding/baiting restrictions, deer rehabilitation bans.

2. For most wildlife management agencies, there will inevitably be a need to communicate to the public, administrators, and key stakeholders, such as legislators and hunters, about specific monitoring approaches and results. It is worth keeping in mind the need to be able to communicate accurately, but meaningfully, to a diverse audience when making choices about strategies, such as sampling frequency, location, or metrics used.

3. To achieve most goals for monitoring CWD trends, consistent application of a sampling plan over an extended timeframe will be needed. Variation in surveillance methods can make meaningful analysis of results difficult. Managers with funding and staffing responsibilities should be aware of and supportive of the need for long-term commitment of resources before a jurisdiction embarks on a CWD monitoring plan.

4. Although commonalities exist, differences between cervid host species and between geographic areas and habitats where CWD occurs likely have significant effects on spatial spread and epidemic dynamics. Choices about goals and strategies for monitoring CWD should be informed by available local information regarding the biology of affected species (for example, recruitment rates, mortality rates, population densities) and cervid landscape use (for example, dispersal, migratory movements, seasonal congregation).

5. As with any large-scale collaborative effort, it is valuable in the formulation of a CWD monitoring plan to be clear about the assumptions underlying design choices. For example, specific to monitoring choices, it is important to acknowledge that there may be limitations in how representative the sample population is to the general population and also limitations in how chosen spatial sampling units represent the heterogeneous landscape.

6. In the course of developing the best monitoring strategies for a jurisdiction, the CWD status, surveillance programs, and management activities of surrounding jurisdictions should be considered. Efforts should be made to share surveillance goals and data among neighboring jurisdictions with a goal of working together to develop regional objectives and monitoring approaches when feasible.

The following sections of this chapter will address concepts and strategies to consider when conducting monitoring surveillance for CWD.

\section{Initial Assessment of a New Chronic Wasting Disease Focus: Evaluating Intensity and Spatial Extent}

Once CWD has been detected in an area where the disease was previously unrecognized, it is important to effectively and efficiently assess disease intensity and spatial distribution to establish a baseline to monitor trends over time. Such an assessment requires defining a biologically relevant susceptible population of one or more host species to survey, choosing a disease-intensity metric (for example, disease prevalence, incidence, or force of infection) with consideration of long-term monitoring goals and desired precision, and choosing a strategy for assessing geographic distribution. Because the territorial ranges of the cervid populations at risk often span jurisdictional boundaries, it may be important to access and incorporate existent knowledge about the CWD status of surrounding jurisdictions in making a decision about assessment approaches. In some circumstances, a regional approach to assessment surveillance may be warranted.

Statistically valid sampling, using either antemortem or postmortem surveillance, is worth striving for to maximize accuracy of the initial disease-intensity estimate. This will facilitate making difficult choices as a jurisdiction embarks on CWD management in a newly identified affected area. It is important to remember that valid methods and sample sizes for disease detection often are not appropriate for establishing the extent and distribution of the disease in a given area or population (see the discussion on sampling sizes later in this chapter for additional information). For example, although "targeted" or "clinical suspect" surveillance is an excellent tool for detecting the disease, it is not an accurate method for determining the intensity of infection within a population, because infected animals are not likely to demonstrate clinical signs until late in the disease course. Likewise, using weighted sampling may be a good tool for assessing spatial distribution but it has limitations for estimating disease intensity. Sampling design, even for initial assessment of disease in a new area, will benefit greatly from input from professionals with experience in biometrics or biostatistics, disease and host ecology, and study design.

Surveillance in the vicinity of a CWD "index case" (defined here as the first case identified in a new area) is an appropriate initial approach for both estimating disease intensity and also initial exploration of the geographic extent 
of disease. However, given that CWD is known to be heterogeneously distributed across landscapes (Joly and Ribic, 2003; Miller and Conner, 2005; Farnsworth and others, 2006; Osnas and others, 2009; Heisey and others, 2010; Walter and others, 2011), representative sampling in all areas of concern will be necessary to achieve the goal of accurately assessing spatial extent and estimating disease intensity in different areas. Therefore, identifying areas of concern [for example, migration corridors (Conner and Miller, 2004), gene flow corridors (Blanchong and others, 2008; Kelly and others, 2010; Cullingham and others, 2011), areas with high cervid habitat abundance (Joly and others, 2006), and refugia (Farnsworth and others, 2005)] will provide a basis for deciding where to sample to make most efficient use of surveillance resources in a short period of time.

Once the initial assessment surveillance is complete, it is probably valuable to revisit disease-management goals before designing an ongoing CWD monitoring program. Although it is desirable to start with a precise estimate of the baseline disease occurrence and distribution, management goals and resource limitations will be important considerations in the degree of certainty needed for these initial disease-assessment estimates. For example, if the goal of disease management is to attempt disease eradication, then a high degree of certainty about both the distribution and extent of infection will be needed to determine if this is a reasonable goal. In contrast, if the management goal is spatial containment of the disease, then having a good estimate of disease distribution may be of primary importance, with perhaps less emphasis on precisely estimating the intensity of the infection within the population. Alternatively, if the goal is limiting the number of new infections within an area where the disease is likely established, then estimating local disease prevalence may be more important than focusing on finding the leading edge of disease spread. Regardless of the management goals, the scope of the problem is defined during the assessment phase, and future monitoring and management decisions will be based upon what is discovered. Therefore, it is worthwhile to devote resources to fully understanding the problem early on for future efforts to be successful.

Once assessment surveillance is completed, ongoing monitoring of an existing focus of CWD will generally include measuring three aspects of the disease: its spatial distribution, its intensity, and how these change over time. Monitoring of spatial distribution and intensity of disease will usually be done simultaneously. However, in specific circumstances, or with limitations in resource availability, the goals of surveillance or management questions may dictate performing either intensity or spatial distribution monitoring alone. These topics are treated separately below to facilitate discussion of each concept, but because they are generally implemented together, there is some necessary redundancy in our coverage of the respective issues.

\section{Monitoring Trends in Intensity of Disease}

One form of disease monitoring is to examine changes or trends in the intensity of disease within a population or region of interest. Tracking fluctuations in disease intensity can be used to answer questions regarding rates of change in disease dynamics or parameters. Before embarking on a monitoring program for investigating disease intensity, it is essential to establish the purpose of the monitoring program or, in other words, what information an agency hopes to gain or what questions need to be answered through this program. If this critical step is foregone, the monitoring program likely will be ineffective and may result in the waste of resources. Thus, framing questions is the first and most important step of developing a monitoring program. Once some direction has been provided, then various metrics, strategies and protocols for monitoring of disease intensity can be more fully considered.

\section{Relevant Questions}

Strategies for evaluating the intensity of disease within populations depend largely on the question(s) monitoring is intended to explore. Such questions might include:

- How much disease is present in the target population?

- How is the disease intensity changing over time?

- How rapidly are CWD-negative animals becoming infected in the target population?

- Is disease intensity changing in response to CWD management actions?

\section{Metrics for Monitoring Trends in Intensity}

A variety of epidemiological metrics exist for measuring the occurrence of diseases in populations. Many of these can be used to express the intensity of CWD in a given population, and when recorded over time, they can be used to monitor trends in CWD occurrence. As noted in the introduction, the specific aims of the monitoring program will dictate to a great degree which of the metrics is chosen. If an agency has modest objectives and needs only to convey the most basic information on the state of the disease to the public, and is willing to accept the severe limitations (and perhaps consequences) of using such ambiguous measures, then the number of CWDpositive animals (in aggregate or per some unit of time) may be a sufficient metric. However, more ambitious objectives, such as disease control or addressing specific research questions integrated into the monitoring program, require more precise metrics. As with any scientific measurement, each 
metric noted below has its strengths and weaknesses. These metrics should be considered carefully before implementing a CWD monitoring program.

\section{Number of Chronic Wasting Disease-Positive Animals}

Arguably, the most basic measure of CWD occurrence in an area is the number of animals shown to be infected (that is, "number testing positive"). The principal strength of this metric is its comprehensibility by the media and the lay public. Often, the first piece of information the media wants to know is the number of test-positive animals, and that information is easy to convey. However, unless the number of CWD-positive animals is zero, this metric has little meaning as a stand-alone measure and can be easily misinterpreted and misconstrued. Because this section discusses surveillance in areas where CWD already has been found, the number of positives will, in most cases, be greater than zero, unless infection rates are low or minimal sampling is occurring. This metric has little or no utility for most purposes without reference to the size of the sampled population. For example, the finding of 2 CWD positive animals in Area X has dramatically different interpretations if the sampled population was 5 animals rather than 5,000 .

\section{Prevalence}

Prevalence, a proportion defined as the number of disease-positive animals divided by some reference population, is a measure of the existing reservoir of disease in that population at (or over) a specified period of time (Rothman and Greenland, 1998). The reference population is specified by the investigator. Often, it is specified as the number of animals tested. In general, prevalence is a useful metric, commonly and readily calculated from data generated by standard surveillance programs. It is relatively easily communicated to the media and the lay public. Consistently measured over time, prevalence can be used to track the size of the reservoir of CWD over time, information of intuitive value for management. Statistical tests for trends are available (Margolin, 1988). However, prevalence measures both new and existing cases of disease at a point in time, and so depending on the disease in question, it may not accurately reflect disease dynamics. In general, changes in population-level transmission of disease are reflected more slowly in prevalence than in incidence (see below). Consequently, it may take several monitoring cycles before CWD occurrence is confirmed to be on the rise (or fall), and if the objective of monitoring is to be able to adaptively apply stronger control measures in the face of increasing infection rates, then this time lag can make control more difficult. As with any proportion, prevalence can be unstable and may be imprecise in situations where few animals are sampled. Attention also must be paid to ensuring that the reference populations that compose the denominator are reasonably consistent across sampling periods. Wide variations in sample gender or age composition should be avoided. Also, it is the responsibility of the resource manager or investigator to consider the extent to which the reference population is representative of the general population to which inference is being made. The possibility of bias in the animals selected for testing, as always, must be considered. For example, a sample of hunter-harvested animals may not be representative of the target population (Conner and others, 2000).

\section{Yearling Prevalence}

One principal limitation of prevalence as a metric is that often it is impossible to determine when an animal testing positive contracted the disease. One way around this constraint is to use prevalence in young animals, say yearlings, as a metric of disease occurrence. Because "yearlings" have only been alive for 12-24 months, animals found infected must have been infected during that time period; for samples based on harvest in most North American jurisdictions, "yearling" cervids are usually 15-18 months old. Consequently, yearling prevalence can be used (O'Brien and others, 2006) as a crude metric for rate of new infections (incidence), a potentially valuable quantity epidemiologically (Rothman and Greenland, 1998). The metric may be most useful in settings such as eastern white-tailed deer populations where large numbers of yearlings are readily available for testing by hunter harvest. However, this metric is subject to instability where sample sizes are low and may have little meaning or utility where yearlings are rarely infected with CWD (Miller and others, 2000; Miller and Conner, 2005).

\section{Force of Infection}

The common desire to use readily available age-specific prevalence data to estimate incidence underlies the metric known as force of infection (FOI). Although more explicit mathematical definitions are available (see for example, Caley and Ramsey (2001), Caley and Hone (2002), and Heisey and others (2006)). FOI can conceptualized as the probability over a short period of time that an uninfected animal will become infected. Because it is more sensitive to changes in transmission than aforementioned disease metrics, tracking trends in FOI over time can provide earlier warning of changes that may have bearing on disease-management decisions than trends in prevalence would provide. FOI has some significant mathematical relationships to other quantities that may be useful epidemiologically, such as probability of disease transmission, which is a critical parameter of most epidemiological models, and methods to derive FOI within an epidemiological framework are available elsewhere (Caley and Hone, 2002; Heisey and others, 2006). In addition, good working estimates of FOI can be derived by using only age-specific prevalence data and spreadsheet programs (G. Hickling, University of Tennessee, oral commun). Depending on the duration of disease and the 
amount of disease-associated mortality (Heisey and others, 2006), trends of FOI over time may or may not closely correspond to trends in disease prevalence (D. O'Brien, Michigan Department of Natural Resources, unpub. data). In situations where disease-associated mortality is low, there may be no particular management advantage to using FOI. In general, it is a more difficult metric to explain to the media and the lay public. However, in situations where there is evidence of significant CWD-associated mortality (Miller and Conner, 2005), or in situations where early detection of managementassociated changes in transmission is desired, use of FOI may have advantages over other simpler metrics.

\section{Incidence}

In monitoring human diseases, incidence is typically the epidemiological metric of choice. Incidence may be defined as the number of new cases of disease in a population at risk over a defined period of time (Rothman and Greenland, 1998). Incidence conveys what is happening with respect to the occurrence of disease at any given point in time. It is the most sensitive and commonly used metric of change in disease transmission rate in a population, and it is a true rate, because some unit of time is included in the denominator. In addition, because incidence also accounts for the size of the population at risk, it allows for comparison across populations or within a population through time. For these reasons, it can provide early warning of changes in conditions that may be relevant to disease control when other disease metrics, such as prevalence, are not able to detect any perturbations. However, the use of incidence in animal populations is considerably less straightforward than in human populations. Surveillance systems that do not test the same individuals repeatedly over time commonly cannot distinguish between newly infected individuals and existing cases. Moreover, quantifying a population at risk is problematic, because it is often impossible to determine which animals are susceptible - as opposed to those already infected and that not susceptible - without testing every individual in the population. Incidence may be very useful as a metric in circumstances where a well-defined population is available for repeated testing over time. For example, the incidence metric may be useful in situations, where live animal testing is being utilized to monitor a discrete population. Because some public familiarity exists with the use of incidence in public health, it may be more easily communicated by the media to the general public.

\section{Strategies and Approaches for Intensity Monitoring}

Approaches for monitoring are dependent on the pertinent questions of interest, the system and metric being examined, and available resources. We begin by describing general strategies that agencies can use to conduct monitoring efforts at varying intensity levels. The first and most intensive monitoring strategy we will detail is the monitoring of cohorts of marked individuals through time. Under this strategy, individual animals are randomly selected from the population or population segments of interest, marked, and followed through time with either periodic testing (for example, tonsil or rectal mucosa biopsy) or testing after the mortality of an individual. In addition, new marks are added each year to maintain adequate sample sizes in each population segment for estimating the metric of interest such as incidence or prevalence. This approach requires significant resources; however, it is likely to provide the most precise information on disease dynamics and the effectiveness of control strategies at a fine resolution. This strategy is most appropriate for small populations, populations of high value where precise disease information is required, or in conjunction with research efforts. An example of using this approach is illustrated by a study from Colorado that followed individually marked mule deer through time to investigate the impacts of CWD and associated selective predation on deer survival (Miller and others, 2008). By using this cohort study design, the authors were able to estimate the intensity metrics of both prevalence and incidence rates for their population of interest.

A second strategy that is also intensive is to actively sample individuals at some desired interval (for example, annually) from the population for disease testing. Selection is assumed to follow some probability sampling scheme (for example, random sampling) that will allow unbiased estimation of the desired intensity metrics. The advantage of active sampling techniques is that they can provide less biased estimates of the metrics of interest than convenience or passive sampling, because the sampling probabilities are known and are under the control of agency personnel. Several approaches have been developed for active sampling, but it is important to keep in mind that they still have some limitations in terms of achieving a completely non-biased approach if underlying assumptions are violated. For example, in areas where road networks provide good spatial coverage of a region, roadkilled individuals may actively be collected and entered into the surveillance stream to monitor CWD on the landscape. However, if road networks are not evenly located across the habitat of the species of interest to account for a heterogeneous distribution of CWD on the landscape, then estimates of intensity metrics may be biased. In addition, the intensity of CWD in road-killed animals may not be representative of CWD in the population of interest (Krumm and others, 2005). Therefore, a thorough understanding of the population of interest, the underlying assumptions, and the biological system are critical when instituting an active monitoring approach. One common approach to active CWD monitoring is random culling of individuals. Although culling is generally implemented as a disease-control mechanism, it can also be simultaneously used as a form of active sampling for disease monitoring if selection of individuals is not targeted. Several studies have used culled individuals to successfully estimate diseaseintensity metrics, most commonly prevalence (Joly and others, 2003; Conner and Miller, 2004; Miller and Conner, 2005; 
Conner, Miller, and others, 2007; Shelton and McDonald, 2011). However, culling, in addition to requiring significant resources, has the added difficulty of potential socio-political repercussions.

A third strategy is to collect individual samples by using passive sampling. Passive sampling is the least field-intensive monitoring tactic. Under this approach, samples enter the surveillance stream without any direct collection action by agency personnel. The most common form of passive sampling is disease testing of hunter-harvested animals, and it is the most common form of CWD intensity monitoring employed by wildlife management agencies. In passive sampling, the probabilities of an individual animal being sampled are unknown, and therefore it is generally assumed that the samples are collected in a random fashion and are representative of the population of interest, although this assumption is undoubtedly violated (Conner and others, 2000; Samuel and others, 2003). In addition, many of the risk factors described in Chapter 1 would suggest this assumption is violated to some degree. Violation of this assumption will lead to biased population estimates for the metric of interest, with the amount of bias dependent on the relationship between the submission probability and the disease state. For example, hunters may be more likely to harvest CWD-infected animals and to submit animals that obviously appear unhealthy, or conversely they may avoid harvesting obviously unhealthy individuals. Many studies have used passive sampling of hunter-harvested animals to investigate CWD intensity for a variety of purposes in varying biological systems (Conner and Miller, 2004; Farnsworth and others, 2005; Farnsworth and others, 2006; Grear and others, 2006; Joly and others, 2006; Osnas and others, 2009; Heisey and others, 2010; Walter and others, 2011).

Other strategies also can be developed that combine those previously described. Both active and passive sampling can be employed for collecting samples for submission into the surveillance stream; for example, collection of road-killed individuals, culling of suspect animals, and hunter-harvest submissions can be combined and used to calculate metrics of interest. Combination strategies generally bring both the advantages and disadvantages of each of the strategies previously described and are those most commonly employed in CWD monitoring.

Within each of these overarching strategies, various approaches can be taken. For example, in systems consisting of multiple susceptible ungulate species, only one species may be selected for monitoring disease intensity. In this case, the selection of the species should be driven by the social, politi$\mathrm{cal}$, and economic importance of the species, the sensitivity of the species to CWD, the amount of disease variability within the species, and the ability to collect adequate sample sizes to detect changes in the metric of interest with reasonable statistical certainty. The advantage of selecting a single species is that limited resources can be applied to providing the most precise estimates of intensity metrics possible for the species most likely to demonstrate trends, rather than spreading resources over several species with decreases in precision for each species-specific estimate. The disadvantage is that it assumes the system is adequately understood to ensure that monitoring of a single species will adequately reflect the underlying disease dynamics and processes of interest for all species. For instance, in some areas in western North America, the ranges of all four susceptible host species overlap. However, in most (but not all) of these situations, deer (Odocoileus spp.) tend to show higher prevalence than sympatric elk and moose populations. Although such streamlining may seem logical and efficient, it may be difficult or impossible for biological or political reasons to select one single species to monitor.

Similarly, within a species, only certain population segments may be selected for monitoring. Once again, if only one segment is to be targeted for surveillance, the same considerations for disease sensitivity, variability, and sample size apply as described for selecting a single species. For example, given the significantly higher prevalence rate for CWD identified in 2-year and older male deer in several jurisdictions (Miller and Conner, 2005; Grear and others, 2006), a focus on older male deer for monitoring disease-intensity trends could be considered. This could be attractive where there is a premium on controlling surveillance costs and effort over the extended period needed to detect management-related changes. Available sample sizes, however, may be a major limiting factor for such a focused sampling strategy. Moreover, it is important to realize that when using subsets of the population, estimates of intensity metrics only apply to the segment from which samples were drawn, and inference cannot be made to the entire population unless the probability of an individual animal falling into the particular population segment is known or can be estimated and the variation in disease dynamics between population segments is clearly understood. Further analysis of existent CWD surveillance data sets and experimental implementation of a monitoring plan focused on a population segment like older-age bucks will likely be needed before its utility is better understood.

Monitoring may also be conducted with varying temporal frequency or resolution, for example, on an annual basis, biannual basis, and so forth. In deciding the appropriate lag period between monitoring events, the biology and epidemiology of the species and the disease of interest need to be considered. For example, because the average course of CWD infection in mule deer is about 2-3 years (Williams, 2005), sampling in multiyear intervals (for example, every 2 or 3 years) may be sufficient for demonstrating temporal trends in intensity of infection. One advantage of using periodic over continuous (annual) monitoring is that supporting resources can be distributed on a rotational basis. In the context of distributing the Federal funding that supported CWD surveillance in many jurisdictions, this approach could be used to provide more funds to agencies that are intensively surveying and reduce allocations to the remaining States in "off years." Theoretically, this rotational system would allow an agency to have a more precise estimate of intensity metrics during monitoring 
years because more resources would be available rather than being diluted across years (Appendix 1, fig. 1-1). If adequate monitoring is conducted, this increased precision also may permit the detection of changes or trends slightly more frequently than what would be discernible by using lower-intensity annual surveys (Appendix 1, figs. 1-2 and 1-3). However, producing estimates of trend and its direction or change detection would require more years of monitoring using a periodic monitoring scheme rather than a continuous monitoring program because of the fewer estimates of intensity metrics generated through time (Appendix 1, figs. 1-2 and 1-3). Another consequence of monitoring periodically is that annual intensity estimates will only be available during monitoring years.

This may be problematic if political and constituent pressures dictate that information from monitoring is available annually, but it may be ideal for situations where antemortem sampling or culling are the main sources of monitoring data. Episodic monitoring could present logistical problems: for example, it may be difficult or more costly to mobilize and maintain the needed personnel and infrastructure for sampling harvested animals periodically, as compared to annually. In addition, if an agency is relying on passive sampling, hunter participation may decrease when an annual monitoring program is not in place, and submissions may not return to needed quotas without a concerted public information effort in survey years.

The foregoing is not an exhaustive list of possible CWD monitoring strategies, and there are undoubtedly a myriad of other potentially viable approaches that could be employed. Our purpose here was to describe the advantages and disadvantages of a few of the most common approaches. Ultimately, each agency will develop its own monitoring plan based on its own specific needs. Regardless of the chosen monitoring strategy, several design aspects should be considered. First, the spatial scale of monitoring activities and the sampling scheme for acquiring samples from across the monitoring area should be carefully described. Given the specified spatial scale, many of the design-based sampling techniques described in Chapter 2 for disease-detection surveillance provide a useful framework for obtaining the needed spatial samples in a rigorous manner. Secondly, within this spatial sampling framework, the methods for acquiring samples from individual animals should be carefully defined and should be based on a probabilistic sampling approach to generate statistically useful estimates of intensity metrics. In addition, at both stages of the sampling procedure, consideration of the population to which the intensity metrics apply is critical. We strongly recommend that responsible agency personnel meet with a statistician or biometrician prior to implementing any monitoring scheme to ensure data are gathered in a manner that assures statistically rigorous estimation of the selected intensity metrics, so that these data can be used to inform management programs and compare trends across jurisdictions.

\section{Where and How Many to Sample for Intensity Monitoring}

Common problems encountered when designing a disease-monitoring program involve determining where and how many individuals to monitor. Unfortunately, as in the foregoing discussion on strategies and approaches, there is no set answer to these questions. The answer once again is dictated by the questions being asked, the system and metrics being studied, and the availability of resources. For example, if the purpose is to detect changes in intensity metrics in the response to control programs, then it may be most appropriate to focus sampling efforts in the vicinity of CWD foci where prevalence rates are the highest and in which changes in intensity are more likely to be detected (see, for example, Conner, Miller, and others, 2007). Conversely, if the interest is in monitoring disease intensity on the "leading edge" of an epidemic, the sampling effort probably should be focused away from foci and instead concentrated in the areas of low disease intensity.

The question of where to sample likely will have a unique solution for each agency; nevertheless, the following four guidelines may be useful for deciding where to monitor. First, monitoring areas should be selected where changes can be detected or trends can be estimated with reasonable precision. Although with infinite sample sizes, theoretically this can be accomplished for any site, in practice, with finite sample sizes changes or trends in intensity, metrics will be most precisely estimated where prevalence is highest. Second, monitoring areas should be established where adequate samples of animals can be collected for disease testing. For example, if an area is largely private land with small deer populations where only a few samples can be collected within the desired timeframe, then this would not be a viable monitoring area, because intensity metrics would vary widely and thereby preclude reliable change or trend estimation. Third, monitoring areas should be selected based on the importance of changes/trends in disease intensity for that region. For example, if disease-control activities have been initiated in a locale and agencies are interested in determining if control efforts are having the desired effect, then monitoring changes/ trends in this area is a high priority, and it should be selected for monitoring. Spatial risk factors as previously described may be used to aid in determining the location of monitoring areas. For example, if CWD is first discovered in a captive cervid facility within a jurisdiction, then monitoring efforts may be focused initially in the vicinity of this facility. Fourth and finally, resources are an important factor for determining regions to monitor. For example, long-term monitoring of a core disease area may require the bulk of the available surveillance resources, thereby limiting monitoring of CWD in other regions.

Once monitoring areas have been established, the next step is to determine how many samples are required from each area. It is critical to note that sample size formulas used for 
detection surveillance are not applicable to disease monitoring! Sample size requirements for disease monitoring are determined by asking the question, "What is the desired size of change in the intensity metric to be detected at a given precision level?" For example, an agency's managers might want to be able to detect with 90 -percent confidence that a 25 -percent change in CWD prevalence will, hopefully, result from culling over the next 5 years. Once the question is framed, a probability model may be assumed depending on the metric of interest; provided appropriate simplifying assumptions are made, this will allow sampling theory to be used to provide insight into the number of samples needed. For interested readers, Lohr (1999) provides an in-depth look at sampling theory and sample size calculation. In addition, for more complex problems that may be encountered in wildlife disease monitoring, computer simulation experiments as described in the previous section also can be used to determine adequate sample sizes to achieve changed detection at the desired precision level. Because of the complexity, we highly recommend at this stage that a statistician or biometrician be consulted to ensure data will be collected in a manner to ensure acceptable precision for intensity metrics. Modeling can be particularly useful in understanding trade-offs between duration, intensity, and replication of monitoring strategies (Conner, Miller, and others, 2007; Nusser and others, 2008), and the failure to invest in such assessments prior to acting may limit the utility of resulting data and ultimately squander opportunities to advance understanding of CWD dynamics and their responses to management.

\section{Challenges with Intensity Monitoring}

The dynamics of any disease, including CWD, are an interwoven chain of complex processes. The purpose of monitoring disease intensity is to detect important changes in these processes, either due to natural events in the progression of the disease or due to management interventions. Metrics of intensity are mathematical constructs aimed at summarizing available information into understandable forms that can signal such changes. However, as with any simplifying model or summarizing statistic, limitations exist. For example, when tracking prevalence over some sampling unit, prevalence is generally reported for that sampling unit. Thus, prevalence is essentially averaged over the unit and ignores the spatial heterogeneity of disease within the sampling unit (Farnsworth and others, 2006). Therefore, when applying intensity metrics, it is important to understand their limitations as well as the appropriate inference that can be drawn from them. It is also important to anticipate that monitoring to detect trends in infection rates or numbers of infected animals on the landscape, especially as part of an effort to control CWD, will be very challenging and will require many years of effort and the investment of significant resources into the surveillance program.

\section{Monitoring Trends in Spatial Distribution}

Monitoring changes in the spatial distribution of CWD represents an important aspect of CWD surveillance in most jurisdictions where foci have been detected. Detecting natural changes (defined here as having arisen from movements of live, free-ranging animals) in the spatial distribution and extent of a CWD focus may be necessary for providing public information and assessing natural trends or the efficacy of disease management. To separate actual disease spread from sampling artifacts, understanding with some certainty the initial or contemporary geographic extent of CWD among susceptible host species in an area of interest is prerequisite to monitoring subsequent changes in distribution. For example, in assessing either natural trends or the effectiveness of management practices intended to minimize geographic spread, if CWD already occurs more widely than initially believed, then subsequent "discovery" of preexisting foci results from an expansion of sampling activities rather than CWD spread. These "discoveries" can lead managers to overestimate the true rate of natural spread, as illustrated during 2002-3 by the "new" discoveries of CWD foci ascribed by some to be evidence of its "rapid spread," or to underestimate the efficacy of their practices. Alternatively, if surveillance to detect new foci is inadequate, then the failure to detect new foci could lead managers to underestimate the extent of geographic expansion of a focus or to overestimate the efficacy of their control actions. It follows that careful attention to this aspect of spatial monitoring should be part of any comprehensive CWD monitoring or management program.

\section{Relevant Questions}

As with other aspects of surveillance and monitoring discussed in this report, the approaches to and extent of monitoring efforts directed toward describing changes in the spatial distribution of CWD depend largely on the question(s) monitoring is intended to answer. Such questions might include:

- What is the current, annual, and/or cumulative geographic distribution of known CWD cases within a jurisdiction?

- How has the geographic distribution of CWD changed, either naturally, or in response to management over time?

- How rapidly is a CWD focus expanding or shrinking? 


\section{Considerations in Monitoring Spatial Changes}

In designing monitoring programs intended to detect changes in the spatial distribution of CWD, a variety of biological, landscape, and operational aspects should be considered. Many of these are common to considerations underlying the design of surveillance programs for detecting new CWD foci as described in the previous chapters, and readers are referred to those chapters for a more detailed discussion.

The first consideration in detecting spatial distribution changes is developing an adequate understanding of the host species biology and ecology. Because natural changes in the spatial distribution of CWD are likely driven almost entirely by movements of infected hosts, understanding the probabilities, distances, demographics, and habitat or other influences on host movement patterns is essential to devising effective monitoring plans. For example, patterns for the spatial spread of CWD in migratory mule deer in montane habitats (Conner and Miller, 2004) may be less predictable than in non-migratory white-tailed deer in an area of relatively homogeneous mixed hardwood and cropland habitat (Joly and others, 2006; Osnas and others, 2009; Heisey and others, 2010).

Another key component of measuring changes in the spatial distribution of CWD is landscape attributes. These factors may influence not only the movements of infected hosts, but also the ability to acquire diagnostic samples or impose management. For example, public land may be more readily and uniformly sampled than private land in some jurisdictions, but private land refugia could be important in establishing or sustaining new CWD foci (Farnsworth and others, 2006; Walter and others, 2011).

Undoubtedly, the most important factor leading to expansion of CWD is proximity to a CWD source. The risk of exposure is highest for animals that have the potential to interact with infected individuals or environments. Thus, intuitively, areas adjacent to infected free-ranging or infected captive cervid facilities are the regions most likely to become newly infected and may be targeted for monitoring activities.

In addition to spatial and demographic risk factors, another important design consideration for any monitoring program is determining which measure of change in spatial distribution is appropriate. The proper measure should be dictated largely by the question the monitoring is intended to answer. The simplest measure may be a minimum known distribution of CWD cases at various times or a minimum convex polygon (MCP) capturing all known locations. In other cases, more complex measures like total landscape affected (for example, MCP buffered by some distance representing natural movement patterns of infected individuals; Joly and others [2006]), host range(s) affected (Conner and Miller, 2004), or intensity of infection across a landscape (Farnsworth and others, 2006; Joly and others, 2006; Osnas and others, 2009; Heisey and others, 2010) may be needed to provide the information required.

The resolution and intensity are also important factors to address during creation and implementation of monitoring programs. Both the desired spatial and temporal resolution of detectable change will influence the intensity of surveillance needed. As with other surveillance applications, smaller increments of change demand proportionally larger sampling effort to detect with confidence. Given likely limitations on available resources in most jurisdictions, developing realistic expectations for detecting changes in spatial patterns may be a significant challenge. Where available, temporal and spatial intervals derived from biological and epidemiological knowledge (for example, mean dispersal distances, dispersal probability, corridors, prevalence at source, and time from first introduction to reasonable likelihood of detecting a new focus based on projected epidemic dynamics) may be the best basis for making such determinations.

Lastly, other confounding risk factors may exist that play a role in CWD spatial dynamics. Sources of potential CWD exposure not related to natural movements of live, free-ranging animals could confound interpretation of spatial distribution and changes therein. Consequently, the occurrence and distribution of other potential risk factors (for example, game farms, taxidermy operations, wildlife rehabilitation activities, artificial baiting or feeding sites, depots for carcasses of vehicle-killed cervids) should be taken into account when designing monitoring plans.

\section{Metrics for Monitoring Trends in Spatial Distribution}

Depending on the specific aims of the monitoring program, it may be considered important to keep track of where CWD exists on the landscape and how that spatial distribution changes over time. The relatively recent ability to acquire spatially referenced data with ease has made several techniques available for monitoring the spatial distribution of CWD. An entire workshop has already been devoted to details of that topic (Conner, Gross, and others, 2007). That work notwithstanding, some general principals warrant restatement here. In general, diseases are not random processes and they do not distribute randomly on the landscape. Extensive data from multiple settings have shown that CWD is no exception (Joly and others, 2003; Miller and Conner, 2005; Farnsworth and others, 2006; Joly and others, 2006; Blanchong and others, 2008; Osnas and others, 2009; Heisey and others, 2010; Walter and others, 2011). Inferences drawn from the distribution of CWD cases must take into account the distribution of the underlying population on the landscape. Knowledge of that distribution is often a required assumption for valid application of statistical methods used to assess the existence and extent of disease clustering. Consequently, monitoring of spatial distribution of CWD needs to account for landscape features such as habitat, winter ranges, topography, and land use that affect distribution of the underlying cervid population (Farnsworth and others, 2006; Joly and others, 2006; Blanchong and others, 2008; Heisey and others, 2010; Kelly and others, 2010; Walter and others, 2011). 
Consideration also should be given to how CWD may be distributed in particular demographic groups of cervids (for example, specific sex and age classes) that are of greatest interest to a particular monitoring program. Occurrence of CWD has been shown to vary significantly by species, age, and gender (Miller and others, 2000; Miller and Conner, 2005; Grear and others, 2006; Walsh and Miller, 2010). Movements of cervids are known to affect the spatial distribution of CWD on the landscape (Conner and Miller, 2004; Blanchong and others, 2008; Skuldt and others, 2008; Kelly and others, 2010; Cullingham and others, 2011). To the extent that age and sex differences influence such movements (for example, dispersal), monitoring programs may be tailored to gather information on particular groups of animals.

Finally, the sensitivity of spatial resolution of monitoring programs deserves some consideration. Species- and genderspecific behavioral factors influence the occurrence of many diseases, including CWD. Behavioral characteristics that limit the number of contacts between different groups of animals affect the probability of exposure to disease (Schauber and others, 2007; Blanchong and others, 2008; Grear and others, 2010; Kelly and others, 2010; Cullingham and others, 2011). For example, once one member of a matriarchal group of white-tailed deer is infected, the number of repeated direct and indirect contacts among those group members likely increases the probability that they will become infected and subsequently increases the prevalence of CWD in the group. Thus, in the limited geographic area that group occupies, CWD is likely to be much more prevalent than disease-testing results aggregated over a larger geographic area would suggest. The practical difficulties of monitoring such small and constantly shifting groups adequately means that monitoring programs must implicitly recognize their limited ability to detect smallscale differences in CWD occurrence (Blanchong and others, 2008; Cullingham, Nakada, and others, 2011).

\section{Strategies and Approaches for Monitoring Surveillance}

In many respects, detecting changes in the spatial distribution of CWD is a special case of detection designed to uncover new CWD foci, as discussed previously, where the greatest single risk factor is proximity to an established (and reasonably well defined) focus of CWD. Consequently, many of the principles, strategies, and approaches for monitoring programs intended to detect changes in spatial patterns are the same as those already described in the previous chapters. As with other detection-oriented CWD surveillance, some form of ongoing targeted or weighted sampling should be regarded as a minimum foundation for monitoring changes in spatial distribution of established foci. Biologically relevant coverage areas should include likely distances and corridors of dispersal and, where applicable, migration movements beyond the periphery of established foci. Selection or prioritization of target areas also could be influenced by intensity of infection in nearby areas, because the probability of an infected animal moving may be higher from those areas than from those farther away, and type of expected movement, because the probability of one or more infected animals moving would likely be greater with migration (a regular, group event) compared to dispersal (a less regular, individual event) (Miller and Conner, 2005, Skuldt and others, 2008). Random sampling may be of value in augmenting monitoring efforts to detect changes in spatial patterns, and some forms of management such as increased harvest or culling may serve an additional purpose in generating additional random samples. In cases where the entire periphery of an endemic focus cannot be intensively and simultaneously sampled, some form of sampling (for example, systematic sampling) of surrounding areas at biological- or risk-based time intervals may be adequate to detect spatial trends. For example, to achieve the level of sampling needed to detect relevant changes in spatial distribution of disease, given limited resources, Wisconsin has chosen to divide the periphery of its CWD-affected area into sampling regions surrounding its CWD core areas. Intense sampling efforts are rotated between these regions at intervals chosen based on spatial risk assessments using deer landscape genetics and habitat barrier analyses (fig. 3).

\section{Challenges with Spatial Monitoring}

As is the case with finding new CWD foci, detecting expansion of an established CWD focus soon after it occurs will be problematic, because relatively few infected animals are in such areas. Similarly, demonstrating true reduction in the size of an established CWD focus (naturally, or in response to some form of management) will be problematic, because small numbers of infected individuals could remain undetected on the landscape. Detection, assessment, and monitoring are perhaps best viewed as a continuum of surveillance activities. If or when occurrence of CWD within a target population becomes undetectable, ongoing disease monitoring effectively reverts to the disease-detection phase. Particularly with a disease such as CWD, where environmental contamination may play a significant role in disease transmission, demonstrating disease eradication from animal populations will require a combination of disease-detection efforts that are intensive (at least at intervals), sustained over time, or both. Attaining a high degree of confidence that CWD is absent from the landscape will likely be a much more difficult matter. However, to achieve management goals in particular areas, such as the desire to remove an area from CWD-related restrictions (for example, deer feeding ban, deer rehabilitation ban, carcass movement restrictions), managers may choose to accept some level of probability of disease eradication as being close enough, and accept some level of risk, especially the risk that CWD transmission is still possible from an environmental reservoir. 


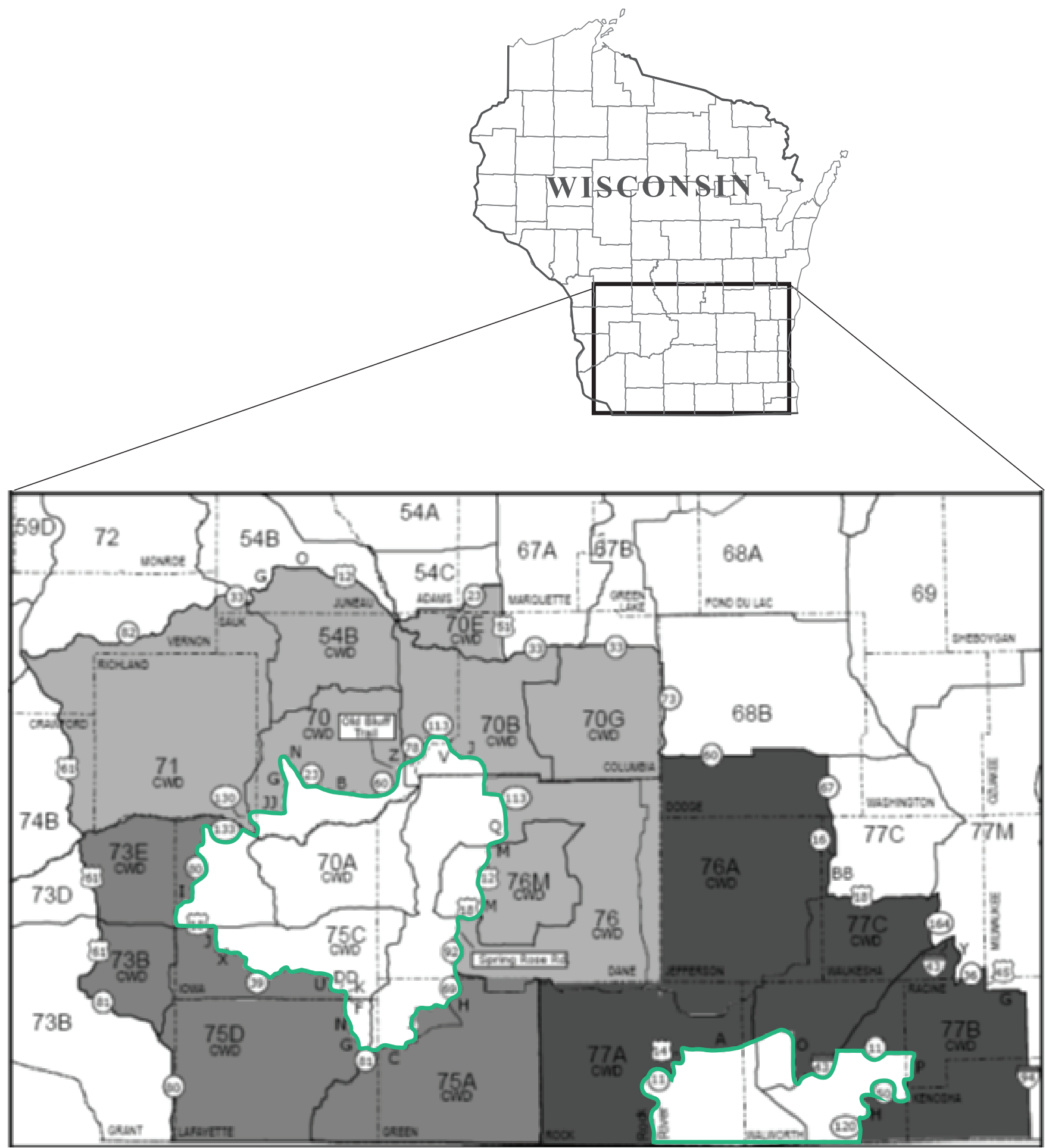

\section{EXPLANATION}

Area 1-Sample 2006

Area 2-Sample 2007

Area 3-Sample yearly
Chronic Wasting Disease Core Areas (Sample yearly)

\author{
County boundary
}

Deer Management Unit boundary

Figure 3. Sampling regions for detecting changes in spatial distribution of chronic wasting disease in Wisconsin during 2006-7. 


\section{Research and Chronic Wasting Disease Monitoring Surveillance}

There are many advantages to considering combined development of a CWD monitoring and epidemiologic research program. There are significant opportunities to use data collected in CWD monitoring surveillance to address important CWD research questions: for example, defining patterns and rates of transmission in different species and in different places, evaluating how landscape features may affect geographic spread, or investigating what role different age or sex cohorts play in epidemiology. In addition, development and adaptation of a monitoring plan will be greatly enhanced by incorporating the results of epidemiologic research, especially focused analyses using regional surveillance data. Though agencies, of course, need to make choices about how limited funds and staff time will be used for CWD surveillance, do not underestimate the value of implementing from the beginning a synergistic CWD monitoring and management-oriented research plan.

One important consideration in planning research using monitoring surveillance data is the quality of the data and its applicability for the intended research questions. The use of opportunistically collected CWD data, for example data derived from hunter service testing programs, may be difficult and very possibly misleading in investigating many CWD research questions. Although the resources available will often be a limiting factor, in general, consider trying to collect as much demographic and spatial data associated with CWD samples as possible. The ideas below are suggestions for beginning research plans; however, they are not an exhaustive list, and others may be more appropriate for a particular situation.

\section{Research That Could Improve Planning of Monitoring Surveillance}

- Use of real CWD datasets to identify which metrics, possibly those applicable for monitoring trends in intensity of disease, are most appropriate to detect disease trends, especially disease trends associated with control actions.

- Use of existent datasets to evaluate what might be appropriate intervals between sampling to achieve specific monitoring surveillance goals, for example, how do prevalence estimates based on data collected every 2-3 years (or every 6 years?) compare to data collected on an annual basis to detect trends in intensity of disease?
- Investigation of how landscape or cervid biology attributes affect spatial spread of CWD in different habitats and with different species (for example, elk) to help refine where monitoring for spatial change may be most important.

- Experimental investigations of if CWD susceptibility changes with age or is different between sexes.

- Analysis of epidemiologic information from cervid facilities (for example, elk or deer farms) within an experimental framework to help estimate parameters such as transmission rates.

\section{Research Ideas to Consider Incorporating With a Monitoring Plan}

- Evaluating age and/or sex differences in disease prevalence, which could assist with focusing sampling for further monitoring or perhaps targeting disease-control efforts.

- Evaluating spatial patterns or spatial "hot spots" of CWD on the landscape, which could assist with identifying areas for focused monitoring for evaluating prevalence trends or identifying areas for enhanced control efforts.

- Collecting data to populate parameters, such as transmission rates and the patterns of transmission in epidemiological models.

- Identifying "matched" treatment and control areas for CWD management efforts, so that scientific evaluation of the efficacy of control efforts is feasible.

\section{Conclusion}

Throughout this chapter we have presented a range of ideas and concerns associated with conducting CWD surveillance after the disease has been detected on the landscape. We have highlighted some of the main issues that arise when managers are faced with the challenges of disease monitoring and hoped to illuminate potential alternatives based on collective experience, epidemiological theory, and our current understanding of CWD disease dynamics. Although the topics addressed herein are not exhaustive with regards to the implementation of a CWD monitoring program, we believe they provide a sound foundation upon which managers can build an effective CWD surveillance program. 


\section{Literature Cited}

Blanchong, J.A.; Samuel, M.D.; Scribner, K.T.; Weckworth, B.V.; Langenberg, J.A.; and Filcek, K.B., 2008, Landscape genetics and the spatial distribution of chronic wasting disease. Biology Letters, v. 4, p. 130-133.

Caley, P., and Ramsey, D., 2001, Estimating disease transmission in wildlife with emphasis on leptospirosis and bovine tuerculosis in possums and effects of fertility control: Journal of Applied Ecology, v. 38, p. 1362-1370.

Caley, P., and Hone, J., 2002, Estimating the force of infection: Mycobacterium bovis infection in feral ferrets Mustela furo in New Zealand: Journal of Animal Ecology, v. 71, p. 44-54.

Conner, M.M.; Gross, J.E.; Cross, P.C.; Ebinger, M.R.; Gillies, R.R.; Samuel, M.D.; and Miller, M.W., 2007, Scaledependent approaches to modeling spatial epidemiology of chronic wasting disease: Salt Lake City, Utah Division of Wildlife Resources, 68 p., accessed Sept. 2, 2011, at http://www.cwd-info.org/index.php/fuseaction/resources. newresearch.

Conner, M.M.; McCarty, C.W.; and Miller, M.W., 2000, Detection of bias in harvest-based estimates of chronic wasting disease prevalence in mule deer: Journal of Wildlife Diseases, v. 36, p. 691-699.

Conner, M.M., and Miller, M.W., 2004, Movement patterns and spatial epidemiology of a prion disease in mule deer population units: Ecological Applications, v. 14, p. 1870 1881.

Conner, M.M.; Miller, M.W.; Ebinger, M.R.; and Burnham, K.P., 2007, A meta-BACI approach for evaluating management intervention on chronic wasting disease in mule deer: Ecological Applications, v. 17, p. 140-153.

Cullingham, C.I.; Nakada, S.M.; Merrill, E.H.; Bollinger, T.K.; Pybus, M.J.; and Coltman, D.W., 2011, Multiscale population genetic analysis of mule deer (Odocoileus hemionus hemionus) in western Canada sheds new light on the spread of chronic wasting disease: Canadian Journal of ZoologyRevue Canadienne De Zoologie, v. 89, p.134-147.

Farnsworth, M.L.; Hoeting, J.A.; Hobbs, N.T.; and Miller, M.W., 2006, Linking chronic wasting disease to mule deer movement scales: A hierarchical Bayesian approach: Ecological Applications, v. 16, p. 1026-1036.

Farnsworth, M.L.; Wolfe, L.L.; Hobbs, N.T.; Burnham, K.P.; Williams, E.S.; Theobald, D.M.; Conner, M.M.; and Miller, M.W., 2005, Human land use influences chronic wasting disease prevalence in mule deer: Ecological Applications, v. 15 , p. $119-126$.
Grear, D.A.; Samuel, M.D.; Langenberg, J.A.; and Keane, D., 2006, Demographic patterns and harvest vulnerability of chronic wasting disease infected white-tailed deer in Wisconsin: Journal of Wildlife Management, v. 70, p. 546-533.

Grear, D.A.; Samuel, M.D.; Scribner, K.T.; Weckworth, B.V.; and Langenberg, J.A., 2010, Influence of genetic relatedness and spatial proximity on chronic wasting disease infection among female white-tailed deer: Journal of Applied Ecology, v. 47, p. 532-540.

Heisey, D.M.; Joly, D.O.; and Messier, F. ,2006, The fitting of general force-of-infection models to wildlife disease prevalence data: Ecology, v. 87, p. 2356-2365.

Heisey, D.M.; Osnas, E.E.; Cross, P.C.; Joly, D.O.; Langenberg, J.A.; and Miller, M.W., 2010, Linking process to pattern: estimating spatiotemporal dynamics of a wildlife epidemic from cross-sectional data: Ecological Monographs, v. 80, p. 221-240.

Joly, D.O., and Ribic, C.A., 2003, Chronic wasting disease in free-ranging Wisconsin white-tailed deer: Emerging Infectious Diseases, v. 9.

Joly, D.O.; Ribic, C.A.; Langenberg, J.A.; Beheler, K.; Batha, C.A.; Dhuey, B.J.; Rolley, R.E.; Bartelt, G.; Van Deelen, T.R.; and Samuel, M.D., 2003, Chronic wasting disease in free-ranging Wisconsin white-tailed deer: Emerging Infectious Diseases, v. 9, p. 599-601.

Joly, D.O.; Samuel, M.D.; Langenberg, J.A.; Blanchong, J.A.; Batha, C.A.; Rolley, R.E.; Keane, D.P.; and Ribic, C.A.; 2006, Spatial epidemiology of chronic wasting disease in Wisconsin white-tailed deer: Journal of Wildlife Diseases, v. 42 , p. $578-588$.

Kelly, A.C.; Mateus-Pinilla, N.E.; Douglas, M.; Brown, W.; Ruiz, M.O.; Killefer, J.; Shelton, P.; Beissel, T.; and Novakofski, J., 2010, Utilizing disease surveillance to examine gene flow and dispersal in white-tailed deer: Journal of Applied Ecology, v. 47, p. 1189-1198.

Krumm, C.E.; Conner, M.M.; and Miller, M.W., 2005, Relative vulnerability of chronic wasting disease infected mule deer to vehicle collisions: Journal of Wildlife Diseases, v. 41 , p. 503-511.

Lohr, S.L., 1999, Sampling design and analysis: Pacific Grove, Calif., Duxbury Press.

Margolin, B.H., 1988, Test for trend in proportions, in Klotz, S., and Johnson, N., (eds.), Encyclopedia of statistical science: New York, Wiley Bros., p. 334-336.

Miller, M.W., and Conner, M.M., 2005, Epidemiology of chronic wasting disease in free-ranging mule deer: spatial, temporal, and demographic influences on observed prevalence patterns: Journal of Wildlife Diseases, v. 41, p. 275-290. 
Miller, M.W.; Swanson, H.M.; Wolfe, L.L.; Quartarone, F.G.; Huwer, S.L.; Southwick, C.H.; and Lukacs, P.M., 2008, Lions and prions and deer demise: PLoS ONE, v. 3, no. 12, e4019, http://dx.doi.org/10.1371/journal.pone.0004019.

Miller, M.W.; Williams, E.S.; McCarty, C.W.; Spraker, T.R.; Kreeger, T.J.; Larsen, C.T.; and Thorne, E.T.; 2000, Epizootiology of chronic wasting disease in free-ranging cervids in Colorado and Wyoming: Journal of Wildlife Diseases, v. 36, p. 676-690.

Nusser, S.M.; Clark, W.R.; Otis, D.L.; and Huang, L., 2008, Sampling considerations for disease surveillance in wildlife populations: Journal of Wildlife Management, v. 72, p. $52-60$.

O’Brien, D.J.; Schmitt, S.M.; Fitzgerald, S.D.; Berry, D.E.; and Hickling, G.J., 2006, Managing the wildlife reservoir of Mycobacterium bovis: The Michigan, USA, experience: Veterinary Microbiology, v. 112, p. 313-323.

Osnas, E.E.; Heisey, D.M.; Rolley, R.E.; and Samuel, M.D., 2009, Spatial and temporal patterns of chronic wasting disease: Fine-scale mapping of a wildlife epidemic in Wisconsin: Ecological Applications, v. 19, p. 1311-1322.

Rothman, K.J., and Greenland, S., 1998, Modern epidemiology: Philadelphia, Penn., Lippincott-Raven, 738 p.

Samuel, M.D.; Joly, D.O.; Wild, M.A.; Wright, S.D.; Otis, D.L.; Werge, R.W.; and Miller, M.W., 2003, Surveillance strategies for detecting chronic wasting disease in free-ranging deer and elk: Results of a CWD surveillance workshop, 10-12 December 2002: U.S. Geological Survey National Wildlife Health Center, 40 p., accessed May 27, 2011, at http://www.nwhc.usgs.gov/publications/fact_sheets/pdfs/ cwd/CWD_Surveillance_Strategies.pdf.
Schauber, E.M.; Storm, D.J.; and Nielsen, C.K.; 2007, Effects of joint space use and group membership on contact rates among white-tailed deer: Journal of Wildlife Management, v. 71, p. 155-163.

Shelton, P., and McDonald, P., 2011, Illinois chronic wasting disease: 2010-2011 surveillance management summary: Illinois Department of Natural Resources. 16 p., accessed Nov. 18, 2011 at http://dnr.state.il.us/cwd/.

Skuldt, L.H.; Mathews, N.E.; and Oyer, A.M., 2008, Whitetailed deer movements in a chronic wasting disease area in south-central Wisconsin: Journal of Wildlife Management, v. 72, p. 1156-1160.

Walsh, D.P., and Miller, M.W., 2010, Designing a weighted surveillance system for chronic wasting disease in Colorado: Journal of Wildlife Diseases, v. 46, p. 118-135.

Walter, W.D.; Walsh, D.P.; Miller, M.W.; Winkelman, D.L.; and Farnsworth, M.L., 2011, Soil clay content underlies prion infection odds: Nature Communications, v. 2, p. 200, http://dx.doi.org/10.1038/ncomms 1203.

Williams, E.S, 2005, Chronic wasting disease: Veterinary Pathology, v. 42, p. 530-549. 


\section{Appendix 1. Periodic Sampling Simulation}

To assess the impacts of periodic sampling on monitoring efforts, we conducted a simple simulation study. We created a homogeneous population of deer in which chronic wasting disease (CWD) was discovered in 5 animals after sampling 250 deer during the first year of a surveillance program. We specified a linear increase in CWD over a 10 -year sampling period with a rate of increase of 0.11 on the logit scale. This resulted in prevalence ranging from 0.02 in year 1 to 0.05 in year 10 . We then randomly sampled from the population, employing two different sampling regimes. In Scenario A, we randomly sampled 100 individuals from the population each year; whereas in Scenario B, we randomly sampled 300 individuals from the population every third year. The total number of individuals tested for disease was identical after 10 years between the two alternative sampling strategies. To determine if an individual selected for sampling was infected with CWD, we generated a random uniform number between zero and one. If this number was less than or equal to the true prevalence, the animal was determined to be CWD positive. This provided the binomial data to estimate prevalence and measure potential disease trends. We examined two simple models: 1) an intercept only model (constant prevalence model) and 2) an intercept model with an additive linear trend (the true model). We use Akaike's Information Criterion (AIC) to compare the models and select the most parsimonious model given the data. We examined associated parameter estimates and 95-percent confidence intervals generated by using PearsonKlopper exact methods. We repeated the simulation for 10,000 repetitions.

We evaluated each scenario to examine its ability to adequately describe the underlying disease process. First, we estimated the mean prevalence under each scenario and compared the mean precision of the estimates averaged across all repetitions. Secondly, we examined the proportion of the simulation repetitions where the true model was correctly selected, and if the true model was selected, we examined the mean estimates of the trend parameter and the associated mean standard 95-percent confidence intervals.
The results from this simulation demonstrated that unsurprisingly during "monitoring years," prevalence estimates under Scenario B were more precise than those employing Scenario A (fig. 1-1). This is due to the larger sample size during monitoring years under Scenario B compared with Scenario A. The simulations also portrayed the difficulty in estimating trends through time due to the binomial variability of the data. Under Scenario A, the correct model was not selected in $\geq 50$ percent of the simulations until year 9 ; whereas under Scenario B, the correct model was not selected in $\geq 50$ percent of the simulations until the final year of monitoring (fig. 1-2). In years where comparisons were possible, the correct model was selected more frequently by employing Scenario B. If the correct model was selected, both Scenarios produced slightly biased trend estimates with both bias and variance of these estimates decreasing through time (fig. 1-3); however, trend parameters were not reasonably estimated (that is, standard errors $<1$ ) until year 5 and year 7 for Scenarios A and $\mathrm{B}$, respectively.

Based on these simple simulation results, it appears that both sampling strategies have strengths and weaknesses. The continuous monitoring strategy provides annual prevalence estimates, and when the correct model is selected, this strategy by virtue of its intensity provides trend estimates sooner than periodic monitoring. In contrast, annual prevalence estimates are available only during select years under the periodic sampling scheme; however, during those monitoring years the larger sample size provides more precise prevalence estimates, leads to selection of the correct model somewhat more often, and estimation of the trend parameter with slightly less bias and more precision. Thus, information about CWD disease dynamics may be gained by using periodic sampling or continuous monitoring strategies, and both alternative sampling strategies potentially have merit based on the needs of the practitioner. 


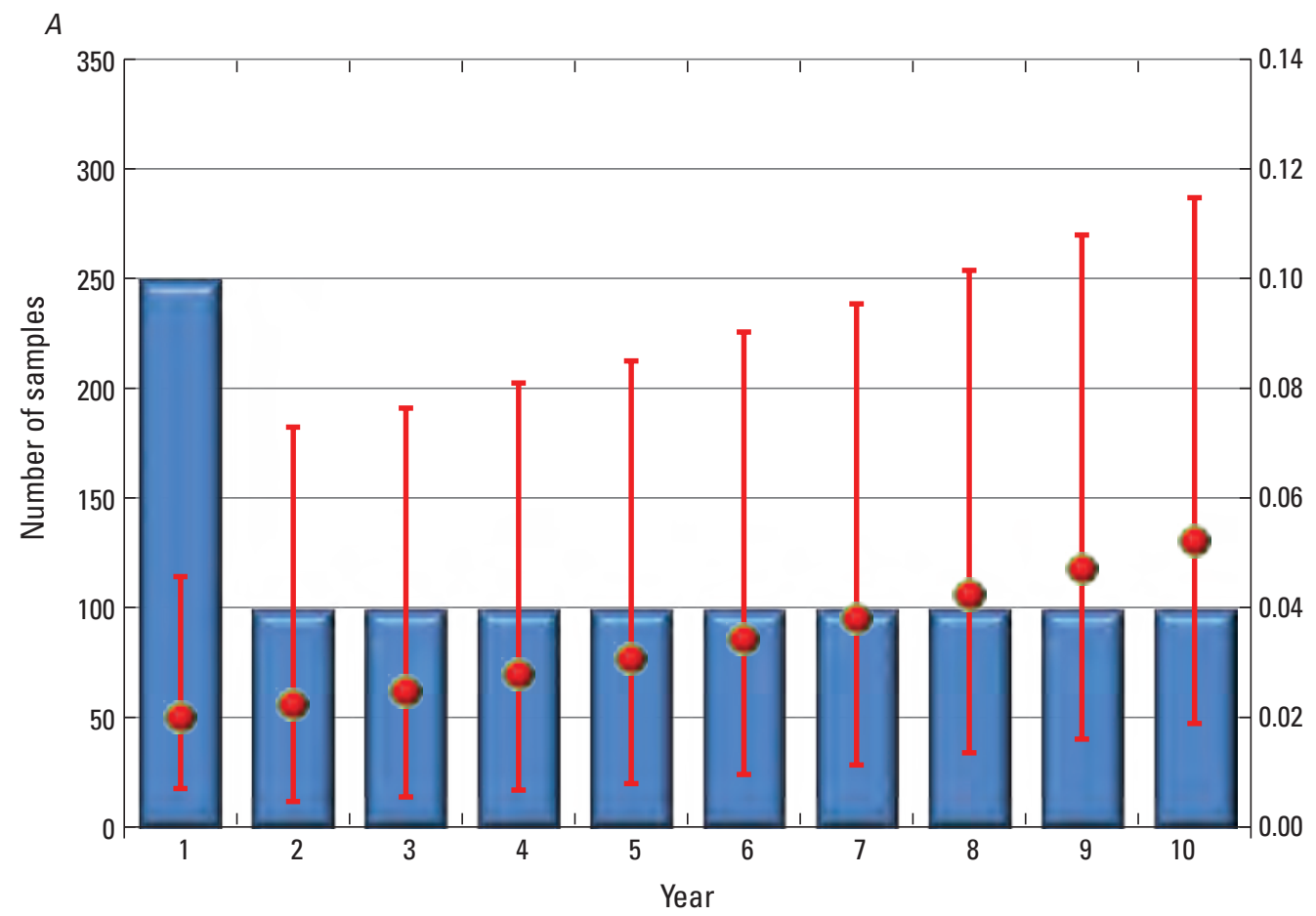

$B$

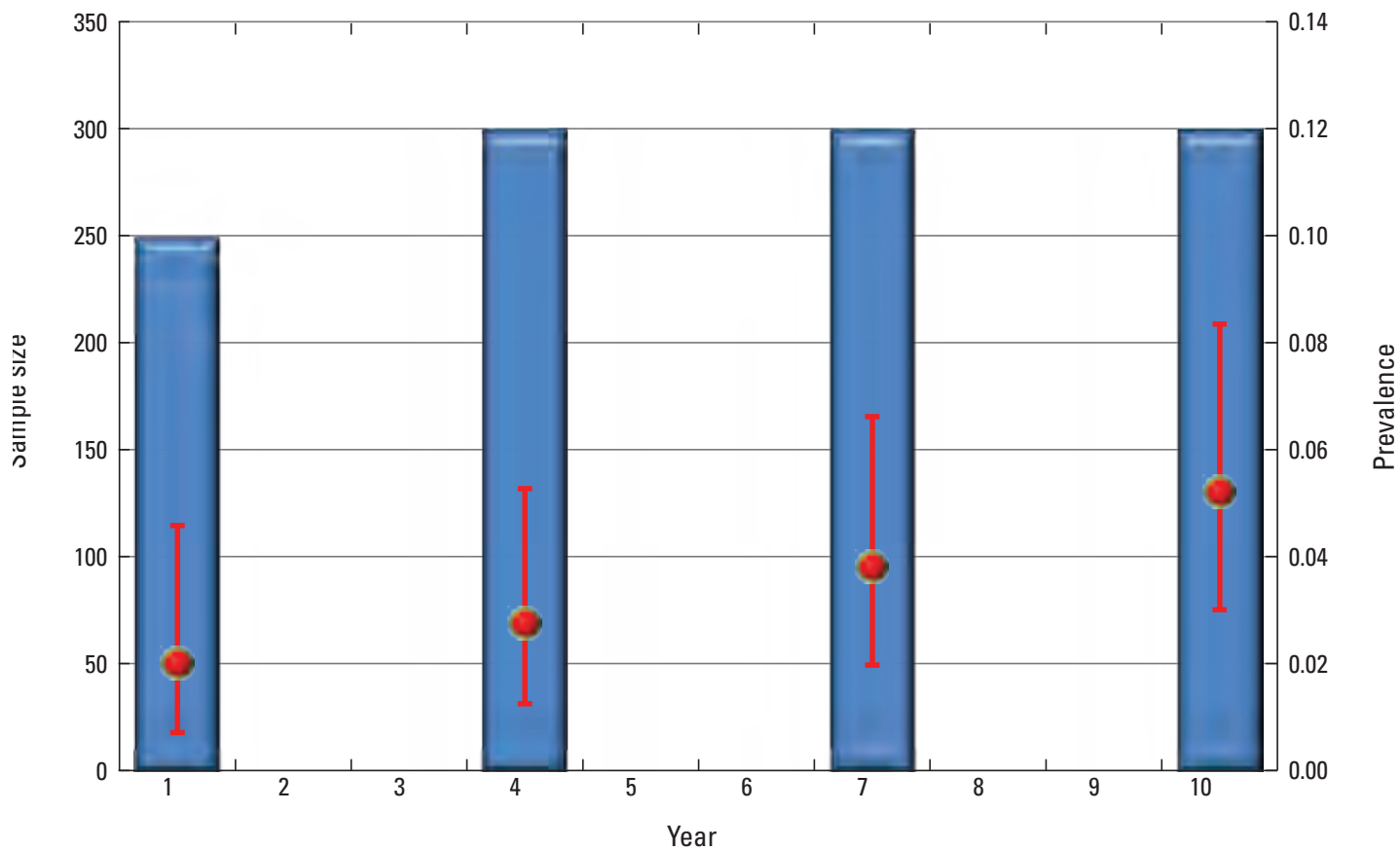

EXPLANATION

Figure 1-1. Comparison of mean chronic wasting disease prevalence estimates and their measures of precision between $A$, Scenario A with continuous disease monitoring and $B$, Scenario $B$ with periodic sampling generated during 10,000 simulation repetitions. Ninety-five percent confidence intervals are based on the Pearson-Klopper method.

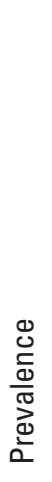

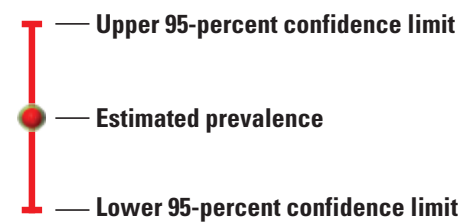




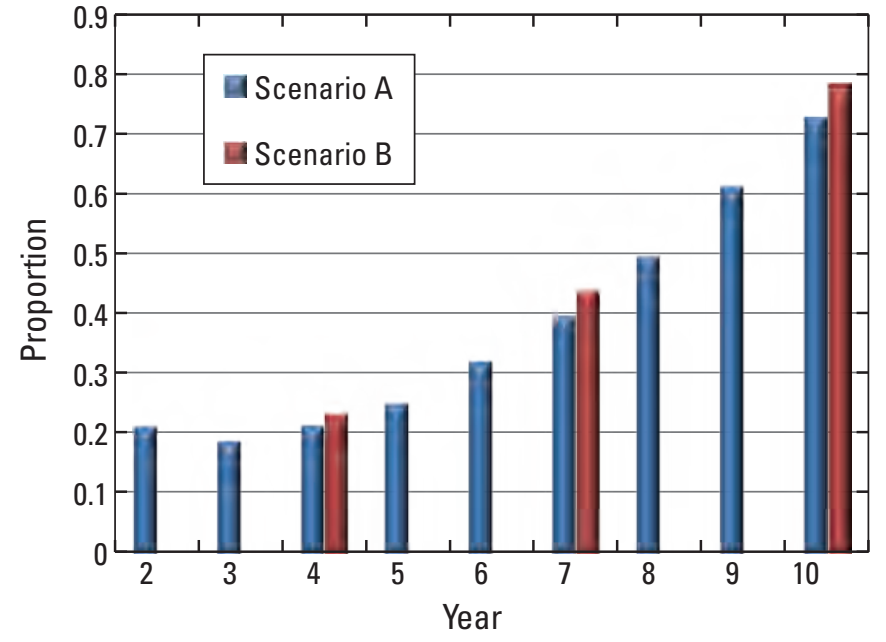

Figure 1-2. Comparison of proportion of 10,000 simulation repetitions where the correct underlying disease model was selected based on Akaike's Information Criterion (AIC) between a continuous sampling regime (Scenario $\mathrm{A}$ ) and a periodic sampling scheme (Scenario B). 

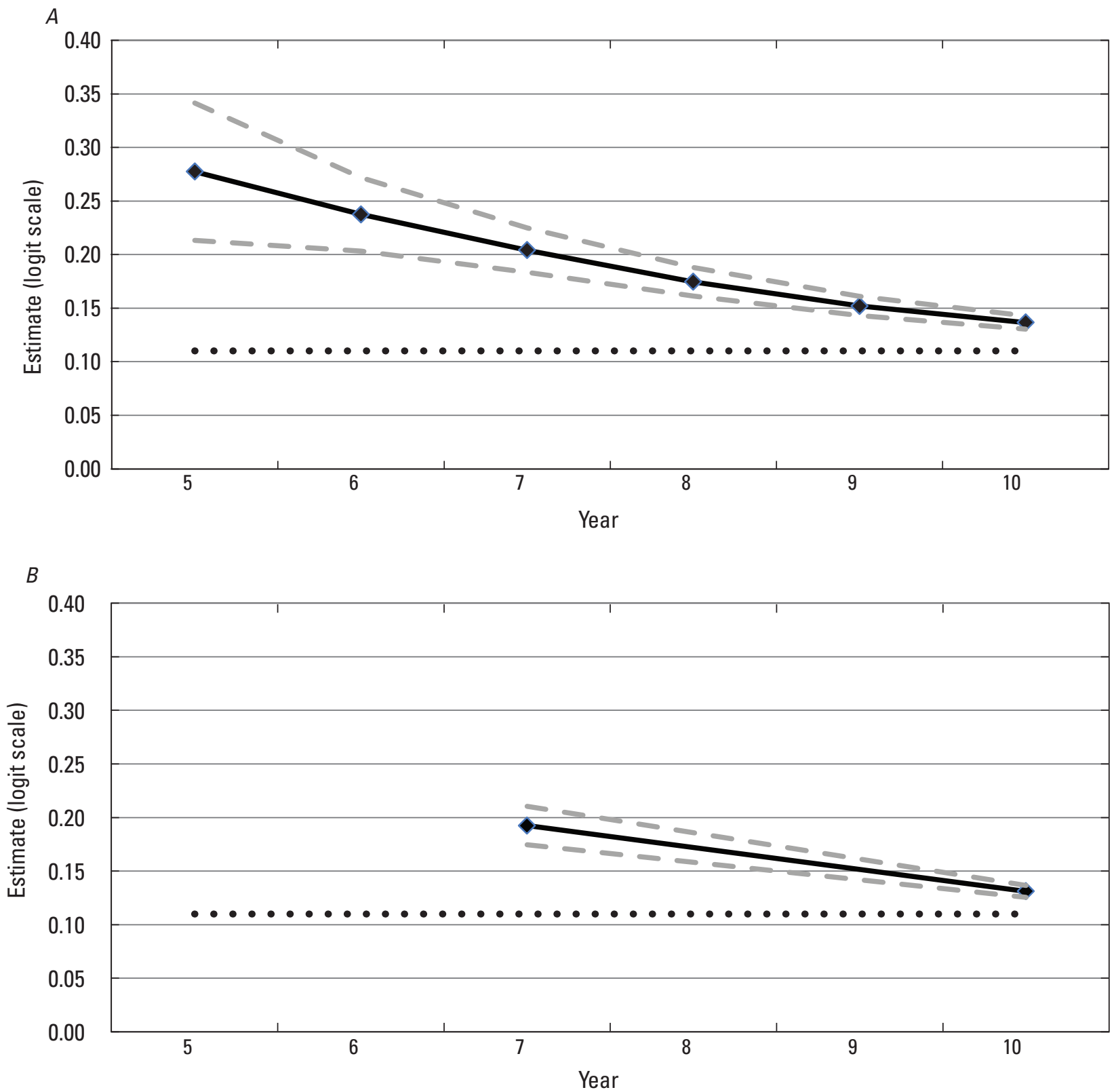

EXPLANATION

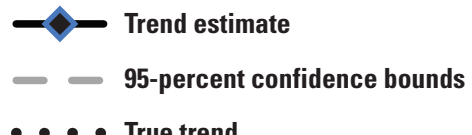

Figure 1-3. Comparison of mean trend estimates and mean, standard 95-percent confidence bounds for models where the correct disease model was selected by using Akaike's Information Criterion (AIC) between $A$, Scenario A with continuous disease monitoring and $B$, Scenario $B$ with periodic sampling (generated during 10,000 simulation repetitions). 

